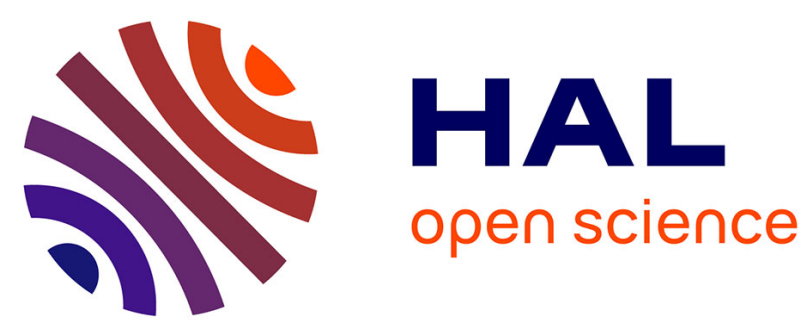

\title{
Implementation of the nudged elastic band method in a dislocation dynamics formalism: application to dislocation nucleation
}

Pierre-Antoine Geslin, Riccardo Gatti, Benoit Devincre, David Rodney

\section{- To cite this version:}

Pierre-Antoine Geslin, Riccardo Gatti, Benoit Devincre, David Rodney. Implementation of the nudged elastic band method in a dislocation dynamics formalism: application to dislocation nucleation. Journal of the Mechanics and Physics of Solids, 2017, 108, pp.49-67. 10.1016/j.jmps.2017.07.019 . hal01569117

\section{HAL Id: hal-01569117 \\ https://hal.science/hal-01569117}

Submitted on 26 Jul 2017

HAL is a multi-disciplinary open access archive for the deposit and dissemination of scientific research documents, whether they are published or not. The documents may come from teaching and research institutions in France or abroad, or from public or private research centers.
L'archive ouverte pluridisciplinaire HAL, est destinée au dépôt et à la diffusion de documents scientifiques de niveau recherche, publiés ou non, émanant des établissements d'enseignement et de recherche français ou étrangers, des laboratoires publics ou privés. 


\title{
Implementation of the nudged elastic band method in a dislocation dynamics formalism: application to dislocation nucleation
}

\author{
Pierre-Antoine Geslin ${ }^{\mathrm{a}, *}$, Riccardo Gatti ${ }^{\mathrm{b}}$, Benoit Devincre ${ }^{\mathrm{b}}$, David Rodney ${ }^{\mathrm{a}}$ \\ ${ }^{a}$ Univ Lyon, Université Claude Bernard Lyon 1, CNRS, Institut Lumière Matière, F-69622, LYON, France \\ ${ }^{b}$ Laboratoire d'Etude des Microstructures, UMR 104 Onera - CNRS, 29 avenue de la division Leclerc, \\ 92322 Châtillon, France
}

\begin{abstract}
We propose a framework to study thermally-activated processes in dislocation glide. This approach is based on an implementation of the nudged elastic band method in a nodal mesoscale dislocation dynamics formalism. Special care is paid to develop a variational formulation to ensure convergence to well-defined minimum energy paths. We also propose a methodology to rigorously parametrize the model on atomistic data, including elastic, core and stacking fault contributions. To assess the validity of the model, we investigate the homogeneous nucleation of partial dislocation loops in aluminum, recovering the activation energies and loop shapes obtained with atomistic calculations and extending these calculations to lower applied stresses. The present method is also applied to heterogeneous nucleation on spherical inclusions.
\end{abstract}

Keywords: Dislocation dynamics, dislocation nucleation, nudged elastic band method, coherency loss

\section{Introduction}

The plastic behavior of metals and alloys depends strongly on temperature because of the role played by thermally-activated processes in dislocation movement. Examples include the nucleation of kink pairs on high Peierls stress dislocations, the cross-slip of screw dislocations and the climb of edge dislocations in creep conditions $[1,2,3]$. The temperature dependence is even stronger in nanostructures, because in absence of bulk sources, dislocations are nucleated from interfaces $[4,5]$, revealing another example of thermally-activated process.

So far, thermally-activated processes involving dislocations have been studied numerically mainly at the atomic scale, using saddle point search methods, such as the nudged elastic band (NEB) method $[6,7,8]$ or the activation-relaxation technique $[9,10,11]$. The case

${ }^{*}$ Corresponding author

Email addresses: pageslin@gmail.com (Pierre-Antoine Geslin), riccardo.gatti@onera.fr (Riccardo Gatti), benoit.devincre@onera.fr (Benoit Devincre), david.rodney@univ-lyon1.fr (David Rodney) 


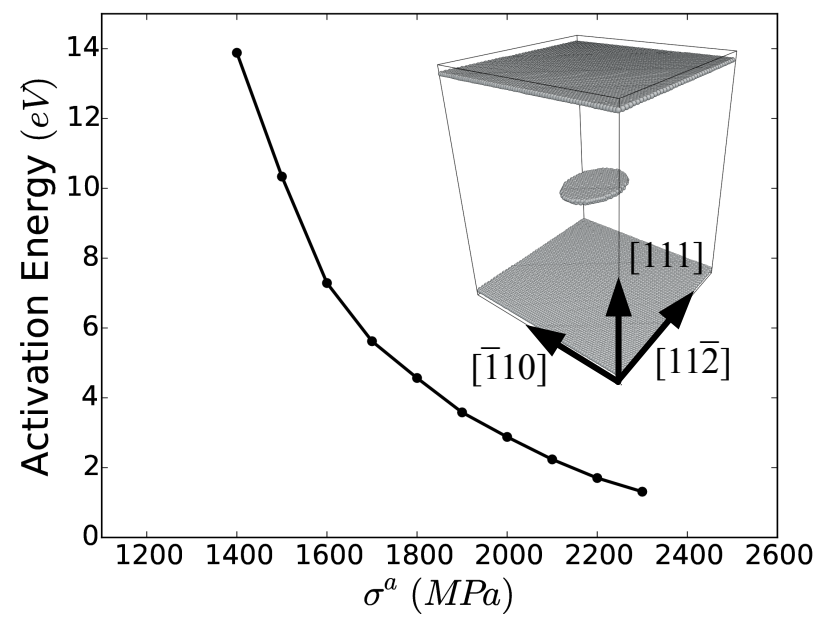

Figure 1: Activation energy function of applied shear stress obtained from atomistic free-end NEB calculations of the homogeneous nucleation of a Shockley partial dislocation loop in aluminum. The inset shows the saddle configuration at an applied shear stress $\sigma^{a}=1.4 \mathrm{GPa}$ (only the defected atoms in the upper and lower free surfaces and in the stacking fault of the loop are shown).

of dislocation nucleation has been particularly studied $[12,13,14,15,16,17]$ to better understand the plastic behavior of nanostructures. As an example, we show in Fig. 1 the activation energy as a function of an applied shear stress computed for the homogeneous nucleation of a Shockley partial dislocation loop in aluminum, modeled with the interatomic potential developed by Mishin et al. [18]. The inset shows the atomistic saddle configuration found at an applied stress $\sigma^{a}=1.4 \mathrm{GPa}$.

The calculations in Fig. 1 used the free-end NEB method, which is briefly recalled here. The degrees of freedom are the atomic positions stored in configuration vectors noted $\boldsymbol{R}$ (of dimension $3 \times N_{a t}$ where $N_{a t}$ is the number of atoms in the system); $E_{a t}(\boldsymbol{R})$ is the corresponding energy. The NEB method consists in building a chain of replicas interpolated between an initial and a final state. In the present case, the initial path is formed of circular loops of increasing radius, created using their elastic displacement field [19]. The replicas are linked between first neighbors using springs in configuration space. The degrees of freedom of a replica $i$ are therefore subjected to (i) the forces $\boldsymbol{F}_{i}^{r}$ derived from the replica potential energy and projected on the hyperplane perpendicular to the chain of replicas and (ii) $\boldsymbol{F}_{i}^{s}$, the contribution of the spring forces associated to the NEB method:

$$
\begin{aligned}
& \boldsymbol{F}_{i}^{r}=-\left(\boldsymbol{\nabla} E_{a t}\left(\boldsymbol{R}_{i}\right)-\boldsymbol{\nabla} E_{a t}\left(\boldsymbol{R}_{i}\right) \cdot \boldsymbol{\tau}_{i}\right) \\
& \boldsymbol{F}_{i}^{s}=k_{N E B}\left(\left|\boldsymbol{R}_{i+1}-\boldsymbol{R}_{i}\right|-\left|\boldsymbol{R}_{i}-\boldsymbol{R}_{i-1}\right|\right) \boldsymbol{\tau}_{i}
\end{aligned}
$$

where $k_{N E B}$ is the elastic constant of the springs between replicas and $\boldsymbol{\tau}_{i}$ the tangent to the path defined using the improved tangent method [20]. A quenched dynamics is used to relax the chain of replicas subjected to the forces in Eqs. (1-2), to obtain the minimum energy 
path (MEP) between the initial and final configurations. For dislocation nucleation, the last replica representing the largest loop is not a local energy minimum. We have therefore used the free-end NEB method [5], where this last image evolves at constant energy, while being subjected to the spring force from its neighboring replica. The replica of maximum energy can be treated differently with the climbing image method [21] to ensure its convergence to the saddle point configuration. The applied stress is controlled by imposing external forces to the atoms within the cut-off radius distance from the upper and lower free surfaces shown in the inset of Fig. 1. The activation energy is then the energy difference between this maximum energy image and the initial configuration. We note that the convergence of the NEB method to a valid minimum energy path relies on the fact that the atomic forces derive from a well-defined energy, i.e. from the variational character of atomistic calculations.

Such calculations are highly informative but are also very limited in term of size scale. The convergence time of the NEB method increases drastically with the number of degrees of freedom (DoFs) in the system, usually limiting its use to volumes smaller than $10^{4} \mathrm{~nm}^{3}$ for dislocation-mediated events. As a consequence, only stresses larger than $1.4 \mathrm{GPa}$ were considered in the example above. This size-scale issue also prevents from modeling complex heterogeneous fields emerging from a surrounding microstructure, for example, of dislocations and/or precipitates. Moreover, atomistic calculations can only give the minimum energy path and activation energy at $T=0 \mathrm{~K}$, while material parameters such as the lattice spacing, elastic constants and stacking fault energy vary significantly with temperature and affect the activation energy, as shown by Ruy et al. [15] in the case of dislocation nucleation. Temperature effects can be included in atomistic saddle-point search calculations but at a large computational cost [22].

It is therefore highly desirable to perform saddle-point searches in higher-scale models, with a limited number of degrees of freedom, to allow for faster calculations in large-scale systems, but also to be able to include temperature effects in an effective way, by varying the model parameters. In this spirit, the NEB method has been incorporated in continuous phase-field models to investigate the nucleation barrier against martensitic transformation [23], shape transitions of vesicles [24] and the wetting of patterned surfaces [25]. This type of mesoscale approaches has also the advantage of averaging out the small energy barriers related to subtle atomic rearrangements, allowing to focus on the most relevant energy barriers for macroscopic phenomena.

For the study of dislocation-mediated mechanisms, generalized Peierls-Nabarro models have been developed to investigate the energetics of dislocation nucleation [26, 27], incorporating the influence of the generalized stacking fault energy and elastic anisotropy. However, these models rely on a short length-scale, representative of the core width and of the same order as the lattice spacing. Therefore, any numerical model used to solve the boundaryintegral equations associated with this approach must rely on a very fine discretization to represent the small length scale, making this approach intrinsically limited to the study of dislocation processes at small length-scales. Sobie and co-workers $[28,29,30]$ have very recently incorporated the NEB method into a dislocation dynamics formalism to investigate the activation energy and attempt frequency of by-pass mechanisms of a dislocation over 
irradiation defects. However, the approach employed by the authors does not rely on a variational framework (the system energy and the forces on the DoF are defined independently from each other), making the application of the NEB method questionable, especially without a careful comparison to a reference problem. In addition, their DD formalism also bears other limitations: it does not account for elastic anisotropy nor does it incorporate the effect of a surrounding precipitate microstructure.

In this article, we propose an implementation of the NEB method in a variational formalism relying on an eigenstrain representation of the dislocations, similar to the one used in the Discrete-Continuous Model [31, 32]. Such an approach is more general than standard DD models because it allows to incorporate straightforwardly the effects of elastic anisotropy and surrounding interfaces, such as grain boundaries, free surfaces, precipitate interfaces. Moreover, it allows for a direct computation of the elastic energy. In addition, we present a methodology to parametrize rigorously the model from atomistic data in order to predict quantitatively activation energies. Dislocation nucleation is considered here as a textbook application of the present approach because this relatively simple process has been extensively studied in the past and can be investigated quantitatively at the same length-scale with atomistic simulations and the continuous approach presented in this article. The article is organized as follows. We first describe the DD framework in section 2. Next, the parametrization of the model on atomistic data is described in section 3 . In section 4 , the parametrized model is used to investigate the homogeneous nucleation of partial dislocations, recovering and extending the results shown in Fig. 1. Finally, in section 5, the model is applied to the more complex case of heterogeneous nucleation on a misfitting precipitate.

\section{Variational dislocation dynamics (VDD) model}

To ensure that the NEB method converges to a valid minimum energy path, the forces on the DoFs of the model must derive from a well-defined potential energy. While this variational property is naturally verified by atomistic calculations, many implementations of DD models are not variational, because the energy of the system is not a physical quantity of great interest during typical DD simulations. For instance, in some DD implementations [33, 34], the dislocation line discretization method makes use of a regularization procedure relying on an interpolation with neighboring segments to calculate a local line tension correction. This correction is necessary, due to the approximation of curved dislocations by straight segments [33], but the resulting force contribution does not derive from a well-defined energy. Moreover, the definition of the DoF in a segment representation of dislocations is not obvious since a configuration is defined by the position and length of the segments, which do not evolve independently from each other.

By way of contrast, nodal DD simulations [35], where the dislocation lines are discretized by connected nodes, are better adapted to NEB calculations because first, the DoFs are the positions of the nodes, which define explicitly and uniquely the dislocation lines. Second, following the work of Cai et al. [36], the nodal forces can be consistently defined in a variational framework using a non-singular elastic representation of dislocations. Here, we will therefore employ a nodal representation of the dislocation lines and extend this approach to 
include core and fault contributions, important to model accurately the nucleation of partial dislocations. Each of these contributions (elastic, core and fault contributions) are described in the following paragraphs.

\subsection{Elastic energy and forces}

\subsubsection{Theory}

The classical analytical theory of dislocations, developed in the linear elasticity framework, describes a dislocation line as a singularity where the stresses and elastic energy diverge [37]. This description, in addition to being unphysical, is not suitable for the NEB method, which needs to rely on a well-defined finite energy. This is why the non-singular description of dislocations introduced by Cai et al. in Ref. [36] is preferred in the present study ${ }^{1}$. In this approach, the Burgers vector density is spread out in the vicinity of the dislocation line. Moreover, we choose an eigenstrain approach as in [32], where dislocation loops are represented as eigenstrain surfaces delimited by the loop contour. From the position of the nodes in the dislocation slip plane, one can build a singular eigenstrain distribution, defined as $\hat{\boldsymbol{\varepsilon}}^{0}(\boldsymbol{r})=\frac{1}{2}(\boldsymbol{b} \otimes \boldsymbol{n}+\boldsymbol{n} \otimes \boldsymbol{b}) \delta(\boldsymbol{r})$ where $\delta(\boldsymbol{r})$ is a Dirac distribution equal to zero outside the planar dislocation loop. To avoid the singular behavior of this description, the eigenstrain is convoluted with the spreading function $w_{a}(\boldsymbol{r})$ [36] characterized by the spreading length $a$ :

$$
\left(w_{a} * w_{a}\right)(\boldsymbol{r})=\frac{15}{8 \pi a^{3}\left[(\|\boldsymbol{r}\| / a)^{2}+1\right]^{7 / 2}}
$$

where the star symbol $*$ denotes the convolution operation and $\|\boldsymbol{r}\|$ is the norm of the position vector $\boldsymbol{r}$. The non-singular eigenstrain is then defined as the convolution $\boldsymbol{\varepsilon}^{0}(\boldsymbol{r})=\left(\hat{\boldsymbol{\varepsilon}}^{0} * w_{a}\right)(\boldsymbol{r})$. This specific form of $w_{a}(\boldsymbol{r})$ leads to simple analytical solutions for the stress field and energy of simple dislocation configurations [36].

Following the seminal work of Mura [39], one can solve the elastic problem associated with an eigenstrain distribution and external boundary conditions (for instance, an applied stress tensor $\boldsymbol{\sigma}_{a}$ ) to obtain the strain field $\boldsymbol{\varepsilon}(\boldsymbol{r})$. Considering the case of homogeneous elastic constants, the elastic energy of the system is:

$$
E_{e l}=\frac{1}{2} \int_{V}\left\{C_{i j k l}\left(\varepsilon_{i j}-\varepsilon_{i j}^{0}\right)\left(\varepsilon_{k l}-\varepsilon_{k l}^{0}\right)\right\} d V-V \sigma_{i j}^{a} \bar{\varepsilon}_{i j},
$$

where $C_{i j k l}$ are the elastic constants and $\bar{\varepsilon}_{i j}=\int_{V} \varepsilon_{i j}(\boldsymbol{r}) d V$, the average of the strain tensor components. The stresses are simply defined as

$$
\sigma_{i j}(\boldsymbol{r})=C_{i j k l}\left(\varepsilon_{k l}(\boldsymbol{r})-\varepsilon_{k l}^{0}(\boldsymbol{r})\right) .
$$

To obtain the stresses acting on the dislocation with a Burgers vector spread out in space, an additional convolution with $w_{a}(\boldsymbol{r})$ is needed and we define the non-singular stresses as $\boldsymbol{\sigma}_{n s}(\boldsymbol{r})=\left(\boldsymbol{\sigma} * w_{a}\right)(\boldsymbol{r})$ and the Peach-Koehler force acting on a point $\boldsymbol{r}$ belonging to a

\footnotetext{
${ }^{1}$ Alternatively the solution proposed in [38] could also have been used.
} 


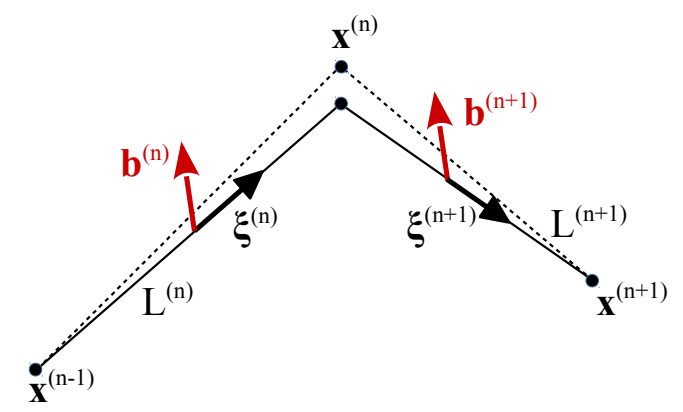

Figure 2: Sketch representing the small displacement of a node.

dislocation segment $(n)$ as $\boldsymbol{f}_{p k}(\boldsymbol{r})=\left(\boldsymbol{\sigma}_{n s}(\boldsymbol{r}) \cdot \boldsymbol{b}^{(n)}\right) \wedge \boldsymbol{\xi}^{(n)}$, where $\boldsymbol{b}^{(n)}$ and $\boldsymbol{\xi}^{(n)}$ are the segment Burgers vector and line vector, respectively. The force on a node $\boldsymbol{x}^{(n)}$ is then obtained from the weighted integration of the Peach-Koehler force (force per unit length) on the adjacent segments of lengths $L^{(n)}$ and $L^{(n+1)}$ (see Fig. 2):

$$
\boldsymbol{f}_{e l}^{(n)}=\int_{L^{(n)}} \frac{u}{L^{(n)}} \boldsymbol{f}_{p k}(u) d u+\int_{L^{(n+1)}} \frac{u}{L^{(n+1)}} \boldsymbol{f}_{p k}(u) d u .
$$

As shown in Ref. [36], this definition of nodal forces satisfies rigorously:

$$
\boldsymbol{f}_{e l}^{(n)}=-\frac{\partial E_{e l}}{\partial \boldsymbol{x}^{(n)}}
$$

therefore ensuring the variational character of the formalism. This property is checked numerically in the next paragraph

\subsubsection{Numerical implementation}

For the sake of generality and to easily include the effects of elastic anisotropy and of a surrounding microstructure, we solve numerically the elastic problem related to the eigenstrain distribution defined above. We note that computing both the elastic forces on the nodes and the elastic energy relies on convolutions, whose exact computation is important to retain the variational character of the model. We therefore use a spectral method, where such operations are simple multiplications in Fourier space. Alternatively, a finite element scheme could be used, as in the discrete-continuum method [31, 32]; although in this case, one has to carefully check that the error associated with the convolution operations performed in real space remains negligible.

The different fields (displacements, stresses,...) are regularly discretized in space and a staggered grid finite difference scheme is employed, where the different components of the displacement field are defined on different grids shifted from one another [40]. We note $d x$ the discretization spacing, taken identical in the three spatial dimensions. This numerical method has been shown to reproduce accurately elastic fields close to sharp variations of the eigenstrain field [41, 42]. 
For simplicity reasons, we consider a planar dislocation loop. For a given position of the nodes, we first initialize the sharp eigenstrain field on the plane of the loop in the region delimited by the nodes. After a Fourier transform and a convolution with $w_{a}(\boldsymbol{r})$, we obtain the corresponding non-singular eigenstrain distribution. Following the resolution method described in Ref. [42], we solve for the displacement field satisfying the elastic equilibrium:

$$
C_{i j k l} \frac{\partial^{2} u_{k}(\boldsymbol{r})}{\partial x_{l} \partial x_{j}}=C_{i j k l} \frac{\partial \varepsilon_{k l}^{0}(\boldsymbol{r})}{\partial x_{j}} .
$$

Once the displacement field $\boldsymbol{u}(\boldsymbol{r})$ is known, deducing the strains and stresses is straightforward. The forces on the nodes are obtained via Eq. (6) involving integration of the PeachKoehler force along adjacent segments. This integration is performed numerically with the stress field at each integration point obtained from a trilinear interpolation between nearest grid points. Finally, the elastic energy of the system is obtained by numerical integration of Eq. (4).

The advantage of the formalism proposed by Cai et al. [36] is to yield analytical solutions for simple dislocation configurations. We first test the computation of the elastic energy by considering a circular dislocation loop of radius $R_{\text {loop }}=10 b$ (shown in the inset of Fig. 3.a) and comparing with the analytical solution [36]:

$$
E_{\text {loop }}=2 \pi R_{\text {loop }} \frac{\mu b^{2}}{8 \pi}\left[\frac{2-\nu}{1-\nu}\left(\ln \left(\frac{8 R_{\text {loop }}}{a}\right)-1\right)+\frac{1}{2}\right]+\mathcal{O}\left(\frac{a^{2}}{R_{\text {loop }}^{2}}\right),
$$

where $\mu$ and $\nu$ are respectively the shear modulus and Poisson's ratio of the material and $b$ the loop Burgers vector. Fig. 3.a shows that the energy obtained numerically converges to the analytical solution of Eq. (9) when the size $L$ of the system is large compared to the loop radius and if $a \gtrsim 3 d x$. For smaller values of $a$, the loop eigenstrain is not well-represented on the finite-difference grid and the energy is larger than expected. In the following, we will systematically use $a \gtrsim 3 d x$ to avoid this numerical artifact.

We also tested the calculation of the forces and the variational aspect of the model by calculating the forces on a 16-node loop in two different ways: (i) numerical integration of the Peach-Koehler force (Eq. (6)), (ii) differentiation of the elastic energy after a small displacement of the position of the nodes. Fig. 3.b shows an excellent agreement between both methods, confirming the variational property of the model. Small discrepancies, barely visible in Fig. 3.b, are attributed to the different approximations used when computing forces and energies: while the stress (and thus the Peach-Koehler force) is interpolated in space from the surrounding grid points, the computation of the energy assumes that the different fields are constant in each voxel.

\subsection{Core energy and forces}

The linear elastic energy introduced in previous section can not account for the high distortions in the dislocation cores and their associated energy. Therefore, we introduce a core energy per unit length denoted $e_{\text {core }}(\theta)$, which depends on the dislocation character, i.e. the angle $\theta$ between the line vector $\boldsymbol{\xi}$ and the Burgers vector $\boldsymbol{b}(\theta=0$ for a screw dislocation). 

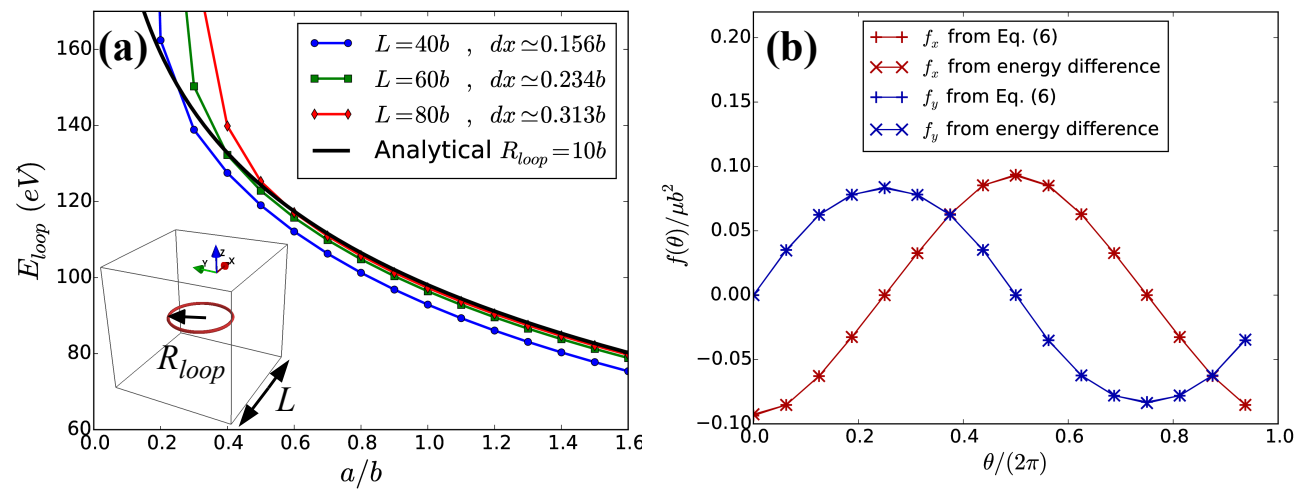

Figure 3: (a) Elastic energy of a circular loop of radius $R=10 b$ as a function of the regularization parameter $a$ and for different system sizes. (b) Comparison of elastic forces acting on the nodes computed in two different ways: from Eq. (6) and from an energy difference after a small displacement.

Let us examine first the symmetry properties that $e_{\text {core }}(\theta)$ must satisfy. A change $\boldsymbol{\xi} \rightarrow-\boldsymbol{\xi}$ does not change the line energy, so $e_{\text {core }}(\theta+\pi)=e_{\text {core }}(\theta)$. Moreover, in the case of face centered cubic (FCC) metals of interest here, $e_{\text {core }}(-\theta)=e_{\text {core }}(\theta)$, due to crystal symmetry. Therefore, we propose to express the core energy with a cosine series:

$$
e_{\text {core }}(\theta)=\sum_{k=0}^{+\infty} a_{k} \cos (2 k \theta)
$$

with $\theta=\arccos \left(\frac{|\boldsymbol{\xi} \cdot \boldsymbol{b}|}{\|\boldsymbol{b}\|}\right)$. This specific form was preferred because it can be conveniently expressed as a polynomial function of the scalar $\alpha=|\boldsymbol{\xi} \cdot \boldsymbol{b}| / \mid \boldsymbol{b} \|$. For example, if we consider only the first three terms of the series $(k=0,1,2)$, we have

$$
e_{\text {core }}(\theta)=c_{0}+c_{1} \alpha^{2}+c_{2} \alpha^{4},
$$

with $c_{0}=a_{0}-a_{1}+a_{2}, c_{1}=2 a_{1}-8 a_{2}$ and $c_{2}=8 a_{2}^{2}$. In the following, we will assume that this truncation is sufficient to describe core energies but we note that including more terms is straightforward.

Let us now consider a node of position $\boldsymbol{x}^{(n)}$ and the adjacent segments of lengths $L^{(n)}$ and $L^{(n+1)}$ (see Fig. 2) with core energies

$$
\begin{aligned}
E_{\text {core }}^{(n)} & =e_{\text {core }}\left(\theta^{(n)}\right) L^{(n)}=\left(c_{0}+c_{1} \alpha_{n}^{2}+c_{2} \alpha_{n}^{4}\right) L^{(n)}, \\
E_{\text {core }}^{(n+1)} & =e_{\text {core }}\left(\theta^{(n+1)}\right) L^{(n+1)}=\left(c_{0}+c_{1} \alpha_{n+1}^{2}+c_{2} \alpha_{n+1}^{4}\right) L^{(n+1)} .
\end{aligned}
$$

where $\alpha_{n}=\left|\boldsymbol{\xi}^{(n)} \cdot \boldsymbol{b}^{(n)}\right| / \| \boldsymbol{b}^{(n)}||$. A small displacement of the node $\boldsymbol{x}^{(n)}$ changes both segment lengths and orientations. The derivatives of $\alpha_{n}$ with respect to the position of the nodes $\boldsymbol{x}^{(n)}$ and $\boldsymbol{x}^{(n+1)}$ are given by 


$$
\begin{aligned}
\frac{\partial \alpha_{n}}{\partial \boldsymbol{x}^{(n)}} & =\frac{\operatorname{sgn}\left(\boldsymbol{\xi}^{(n)} \cdot \boldsymbol{b}^{(n)}\right)}{\left\|\boldsymbol{b}^{(n)}\right\| L^{(n)}}\left(\boldsymbol{b}^{(n)}-\boldsymbol{\xi}^{(n)}\left(\boldsymbol{\xi}^{(n)} \cdot \boldsymbol{b}^{(n)}\right)\right) \\
\frac{\partial \alpha_{n+1}}{\partial \boldsymbol{x}^{(n)}} & =-\frac{\operatorname{sgn}\left(\boldsymbol{\xi}^{(n+1)} \cdot \boldsymbol{b}^{(n+1)}\right)}{\left\|\boldsymbol{b}^{(n+1)}\right\| L^{(n+1)}}\left(\boldsymbol{b}^{(n+1)}-\boldsymbol{\xi}^{(n+1)}\left(\boldsymbol{\xi}^{(n+1)} \cdot \boldsymbol{b}^{(n+1)}\right)\right),
\end{aligned}
$$

and the force acting on a node of position $\boldsymbol{x}^{(n)}$ coming from the core contribution is expressed as:

$$
\boldsymbol{f}_{\text {core }}^{(n)}=-\frac{\partial E_{\text {core }}^{(n)}}{\partial \boldsymbol{x}^{(n)}}-\frac{\partial E_{\text {core }}^{(n+1)}}{\partial \boldsymbol{x}^{(n)}}
$$

where

$$
\begin{aligned}
\frac{\partial E_{\text {core }}^{(n)}}{\partial \boldsymbol{x}^{(n)}} & =\frac{\partial \alpha_{n}}{\partial \boldsymbol{x}^{(n)}}\left(2 c_{1} \alpha_{n}+4 c_{2} \alpha_{n}^{3}\right) L^{(n)}+\left(c_{0}+c_{1} \alpha_{n}^{2}+c_{2} \alpha_{n}^{4}\right) \boldsymbol{\xi}^{(n)} \\
\frac{\partial E_{\text {core }}^{(n+1)}}{\partial \boldsymbol{x}^{(n)}} & =\frac{\partial \alpha_{n+1}}{\partial \boldsymbol{x}^{(n)}}\left(2 c_{1} \alpha_{n+1}+4 c_{2} \alpha_{n+1}^{3}\right) L^{(n+1)}-\left(c_{0}+c_{1} \alpha_{n+1}^{2}+c_{2} \alpha_{n+1}^{4}\right) \boldsymbol{\xi}^{(n+1)} .
\end{aligned}
$$

\subsection{Stacking fault energy and resulting forces}

In the case of the nucleation of partial dislocations, a stacking fault forms inside the loops. As pointed out in Ref. [14], the disregistery across the slip plane (denoted $\zeta$ ) is not necessarily equal to the Burgers vector. Accounting for this effect is important to reproduce quantitatively the activation energy of the nucleation event because in the case of small dislocation loops as for nucleation, the stacking fault energy contribution becomes comparable to the elastic energy. We note $e_{g s f}(\zeta)$ the generalized stacking fault energy (in units of energy per unit area) assumed to be constant in space and to depend only on a scalar displacement jump $\zeta$ in the direction of the partial Burgers vector. For a given loop, the energy contribution from the stacking fault is

$$
E_{s f}=e_{g s f}(\zeta) \mathcal{A}\left(\left\{\boldsymbol{x}^{(n)}\right\}\right)
$$

where $\mathcal{A}\left(\left\{\boldsymbol{x}^{(n)}\right\}\right)$ is the loop area, computed from the nodal positions. We make the simplifying assumption that the core energy does not vary with the amplitude of the disregistery $\zeta$. A small displacement of a node $\boldsymbol{x}^{(n)}$ changes the area of the loop and the resulting force depends on the length and orientation of the neighboring segments:

$$
\boldsymbol{f}_{s f}^{(n)}=-\frac{\partial E_{s f}}{\partial \boldsymbol{x}^{(n)}}=-\frac{e_{g s f}(\zeta)}{2}\left(\boldsymbol{\xi}_{\perp}^{(n)} L^{(n)}+\boldsymbol{\xi}_{\perp}^{(n+1)} L^{(n+1)}\right),
$$

where $\boldsymbol{\xi}_{\perp}^{(n)}$ is normal to $\boldsymbol{\xi}^{(n)}$ and oriented towards the outside of the loop (i.e. $\boldsymbol{\xi}^{(n)} \wedge \boldsymbol{\xi}_{\perp}^{(n)}=1$ with our convention).

The disregistery $\zeta$ is another DoF of the system, which is treated differently from the nodal positions. We assume that its evolution is fast compared to the position of the nodes 
and that it reaches instantaneously an optimum value for which the sum of the elastic and stacking fault energies is minimum:

$$
\min _{\zeta}\left(E_{e l}(\zeta)+E_{s f}(\zeta)\right)
$$

This minimization is straightforward because the elastic contribution depends quadratically on $\zeta$ :

$$
E_{e l}(\zeta)=W_{0}+W_{1} \zeta+W_{2} \zeta^{2}
$$

where $W_{i}$ are constants that depend on the dislocation configuration and can be computed in the general case using volume integrals. In the particular case of an isolated loop, an analytical calculation is possible, yielding:

$$
\begin{aligned}
& W_{0}=-\frac{1}{2} \sigma_{i j}^{a} \varepsilon_{i j}^{a} V \\
& W_{1}=-\left[\sigma_{i j}^{a} \bar{\varepsilon}_{i j}^{0} V\right] / \zeta \\
& W_{2}=\left[E_{e l}(\zeta)+\frac{1}{2} \sigma_{i j}^{a}\left(2 \bar{\varepsilon}_{i j}^{0}+\bar{\varepsilon}_{i j}^{a}\right) V\right] / \zeta^{2}
\end{aligned}
$$

where $\varepsilon_{i j}^{0}=\frac{1}{V} \int_{V} \varepsilon_{i j}^{0} d V$ are the average eigenstrain and $\varepsilon_{i j}^{a}=S_{i j k l} \sigma_{k l}^{a}\left(S_{i j k l}\right.$ are the compliances). We note that the terms $W_{1}$ and $W_{2}$ are independent of $\zeta$ because the terms between square brackets in their expression evolve respectively linearly and quadratically with $\zeta$. Assuming that the form of the generalized stacking fault energy $E_{s f}(\zeta)$ is known, the value of $\zeta$ minimizing the sum $E_{e l}(\zeta)+E_{s f}(\zeta)$ can be easily found.

In this section, we have detailed the different contributions of the energy of the system (the elastic energy, core energy and stacking fault energy), paying a particular attention to the variational character of the model, i.e. the derivation of the forces on the DoFs from the energy. The next step consists in parametrizing carefully these different energy contributions in order to reproduce atomistic calculations.

\section{Parametrization of the model from atomistic simulations}

In order to compare the present variational dislocation dynamics (VDD) with atomistic calculations, the interatomic potential of Mishin et al. for aluminum [18] is considered. This potential is known to reproduce accurately several properties of aluminum, such as the lattice parameter of the stable FCC structure and its elastic constants at $T=0 \mathrm{~K}$ (see Tab. 1). These values of the elastic moduli are used to parametrize the elastic energy. In the following, we will also use the isotropic elastic constants $\mu=28.9 \mathrm{GPa}$ and $\nu=0.34$, obtained from the Scattergood-Bacon average [43, 44], known as the most accurate isotropic average when dealing with the elastic properties of dislocations [2]. 


\begin{tabular}{|c|c|c|}
\hline & From litterature $[45,46,47]$ & EAM potential $[18]$ \\
\hline$a_{0}(\AA)$ & 4.05 & 4.05 \\
$C_{11}(\mathrm{GPa})$ & 114 & 114 \\
$C_{12}(\mathrm{GPa})$ & 61.9 & 61.6 \\
$C_{44}(\mathrm{GPa})$ & 31.6 & 31.6 \\
$U_{s f}\left(\mathrm{~mJ} / \mathrm{m}^{2}\right)$ & $120-144$ & 146 \\
\hline
\end{tabular}

Table 1: Values of the lattice parameter, elastic constants and stacking fault energy from the literature and reproduced with the EAM potential of Mishin et al. [18]
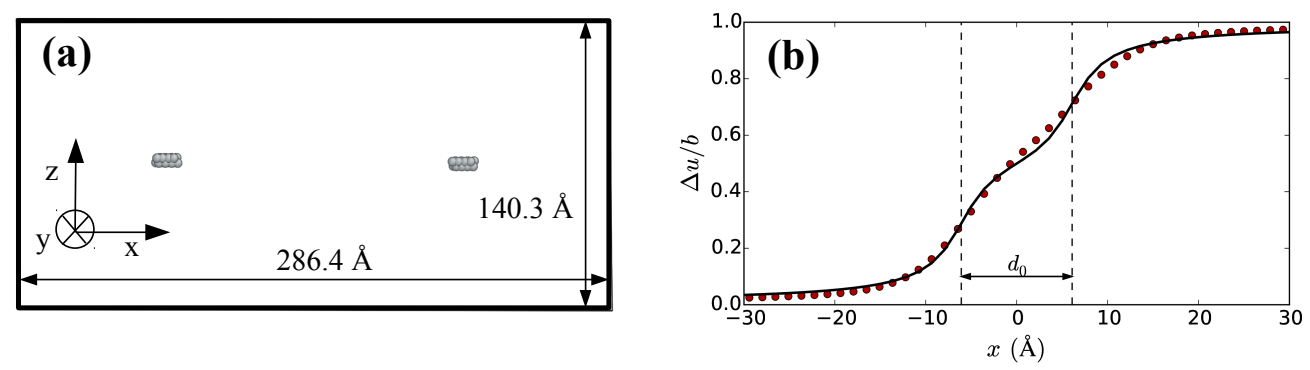

Figure 4: (a) Snapshot of the atomic configuration of the dislocation dipole where only the atoms belonging to the stacking faults are shown. (b) The dissociation distance is computed by fitting the disregistery along the dislocation glide plane against Eq. (26). Atomistic displacement jumps are plotted in red and the fitted function as a continuous black line

\subsection{Core energy of partial dislocations}

In the following, we will be mostly interested in the nucleation of partial dislocations. Then, model parameters used to defined the core energy in Eq. (10) must be calculated to accurately reproduce Shockley partial properties. To extract core energies from atomistic calculations, we first introduce a dipole of perfect edge dislocations into an atomistic simulation cell ${ }^{2}$. The relaxation of the system leads to the dissociation of the perfect dislocations into Shockley partials as shown in Fig. 4.a. The dissociation distance between partials (noted $d_{0}$ ) is extracted from the displacement jumps between the lower and upper atomic planes of the dislocation slip plane. As shown in Fig. 4.b, the atomistic solution is well reproduced with the Peierls-Nabarro model [37]:

$$
\Delta u(x)=\frac{b}{2 \pi}\left(\pi+\arctan \left(\frac{x-d_{0} / 2}{w}\right)+\arctan \left(\frac{x+d_{0} / 2}{w}\right)\right),
$$

using the dissociation distance $d_{0}$ and the core width $w$ as fitting parameters. We display in Tab. 2 the dissociation distance obtained for different dislocation characters $(\phi$ is the angle between the Burgers vector of the perfect dislocation and the dislocation line), which compare well with the results in Ref. [49].

\footnotetext{
${ }^{2}$ We use the software Babel [48] to introduce dislocations in a periodic cell
} 


\begin{tabular}{|c|c|c|}
\hline$\phi$ & $d_{0}(\AA)$ (this study) & $d_{0}(\AA)$ (from $\left.[49]\right)$ \\
\hline 0 (screw) & 6.34 & 6.30 \\
$\pi / 6$ & 8.53 & 7.16 \\
$\pi / 3$ & 10.19 & - \\
$\pi / 2$ (edge) & 12.19 & 12.03 \\
\hline
\end{tabular}

Table 2: Values of the dissociation distance $d_{0}$ obtained from the fitting procedure described herein above and obtained from a slightly different procedure based on the Burgers density [49] using the same atomic potential [18].

To deduce the core energy of partial dislocations, we follow the methodology proposed by Clouet et al. $[50,51,52]$ and decompose the total energy of the atomistic simulation into different contributions. To distinguish them from the energies of the model described in section 2 , the energies associated with atomistic simulations are noted with cursive notation:

$$
\mathscr{E}_{a t}=\mathscr{E}_{e l}\left(r_{c}\right)+\mathscr{E}_{s f}+\mathscr{E}_{\text {core }}\left(r_{c}\right)
$$

We introduce a core radius $r_{c}$ and distinguish the core regions, cylinders of radius $r_{c}$ centered on the partials from the rest of the cell. We choose $r_{c}=b \simeq 2.86 \AA$ but this choice remains arbitrary. The contribution $\mathscr{E}_{e l}\left(r_{c}\right)$ represents the elastic energy computed for a periodic arrangement of singular partial dislocations, excluding the core regions around each partial; this term is calculated within the framework of linear anisotropic elasticity using the work of Clouet et al. [48, 50, 51, 52]. This approach is based on the formalism introduced by Eshelby et al. [53] and extended by Stroh [54]. The conditionally convergent sums over periodic images are regularized based on the work of Cai et al. [55]. We note that core tractions, which result from excluding the core regions for elastic energy computations, are here included and are essential to obtain reliable core energies. $\mathscr{E}_{s f}$ is the stacking fault contribution of the atomistic system containing two stacking faults and is expressed as $\mathscr{E}_{s f}=2 U_{s f} d_{0} L_{\|}$where $L_{\|}$is the dislocation length (i.e. the width of the simulation cell) and $U_{s f}=146 \mathrm{~mJ} / \mathrm{m}^{2}$ (see table 1 ). The core contribution $\mathscr{E}_{\text {core }}\left(r_{c}\right)$ also depends on the choice of $r_{c}$ and is defined as the remaining energy, representative of the large non-linear deformations localized in the core regions.

We note that, similarly to the choice of $r_{c}$, the definition of the stacking fault energy as $\mathscr{E}_{s f}=2 U_{s f} d_{0} L_{\|}$is not unique: it could have been defined with a stacking fault width $d_{0}-2 r_{c}$, i.e. excluding the contribution of the partials core regions. Using the same definitions as above, the core energy would be larger because of the contribution of the stacking fault energy in the core region, but the dislocation behavior would be unchanged, provided that the stacking fault area for a partial dislocation loop is defined consistently by excluding a band of width $r_{c}$ around the dislocation loop.

Applying the above methodology for different dislocation orientations, we compute the core contribution $\mathscr{E}_{\text {core }}\left(r_{c}\right)$ and check that it does not depend on the size of the atomistic cell (see Fig. 5.a). We point out that the character of the partial (denoted $\theta$ ) is different from that of the perfect dislocations $(\phi)$. Therefore, extracting the core energy of the partials as a function of $\theta$ relies on the knowledge of the decomposition of the perfect dislocations [37]. 

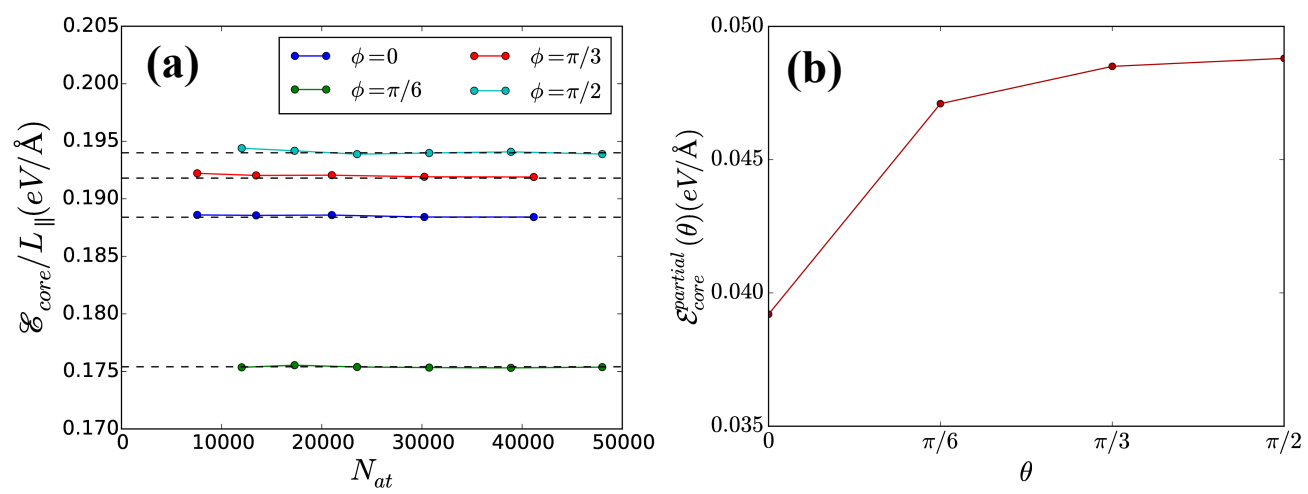

Figure 5: (a) Convergence of energy attributed to the core contribution in the simulated system function of the number of atoms in the system. (b) Core energy of Shockley partial dislocations function of their character.

For the different values of $\phi$, we can then express the core contribution as a function of the core energies of the partials:

$$
\begin{aligned}
& \mathscr{E}_{\text {core }}(\phi=0)=\left(4 \mathcal{E}_{\text {core }}^{\text {partial }}\left(\theta=\frac{\pi}{6}\right)\right) L_{\|} \\
& \mathscr{E}_{\text {core }}\left(\phi=\frac{\pi}{6}\right)=\left(2 \mathcal{E}_{\text {corte }}^{\text {partial }}(\theta=0)+2 \mathcal{E}_{\text {core }}^{\text {partial }}\left(\theta=\frac{\pi}{3}\right)\right) L_{\|} \\
& \mathscr{E}_{\text {core }}\left(\phi=\frac{\pi}{3}\right)=\left(2 \mathcal{E}_{\text {core }}^{\text {partial }}\left(\theta=\frac{\pi}{6}\right)+2 \mathcal{E}_{\text {core }}^{\text {partial }}\left(\theta=\frac{\pi}{2}\right)\right) L_{\|} \\
& \mathscr{E}_{\text {core }}\left(\phi=\frac{\pi}{2}\right)=\left(4 \mathcal{E}_{\text {core }}^{\text {partial }}\left(\theta=\frac{\pi}{3}\right)\right) L_{\|}
\end{aligned}
$$

Inversion of this system of equations yields the core energy of the partials as a function of dislocation orientation. Results of this calculation are plotted in Fig. 5.b.

The next step consists in parameterizing Eq. (10) from the core energy $\mathcal{E}_{\text {core }}^{\text {partial }}(\theta)$. This requires an additional effort since the core energy defined from Eq. (27) accounts from the non-linearities located within the core regions of radius $r_{c}$ within a singular representation of dislocations, while the non-singular approach discussed in section 2.1 assumes linear elasticity everywhere, including the core regions. A way to parametrize the model is to follow Ref. [56] and choose the regularization parameter $a$ such that the elastic energy of the regularized dislocations matches the total energy of the atomistic calculation. Repeating this procedure for different dislocation orientations yields a function $a(\theta)$. However, this approach is not convenient computationally since the convolution operations by $w_{a}(\boldsymbol{r})$ (see section 2.1) are global operations by nature and cannot be restricted to segments of specific orientations. Moreover, this procedure typically yields values of $a$ of the order of $b$ (see Ref. [56]), which would limit the study to small systems.

We therefore follow a different approach by considering $a$ as a numerical parameter chosen such that the total core and elastic non-singular energy matches its singular counterpart. To this end, we consider a (non-atomic) system of width $L_{\|}$where we introduce a dipole of 
straight partial dislocations of orientation $\theta$ in a cell with periodic boundary conditions (no need to consider the stacking fault here). We then compute the anisotropic elastic energy of the system using two methods. First, we consider the partial dislocations as singular defects and exclude cylinders of radius $r_{c}=b$ around them; we note $E_{e l}^{s}\left(r_{c}\right)$ the energy of this singular representation (computed with the software Babel [48]). Second, we consider the non-singular theory of dislocations and compute numerically the elastic energy $E_{e l}^{n s}(a)$ with the present elastic solver for different values of $a$. The 'non-singular' core energy (per unit of length) is then defined as the difference:

$$
e_{\text {core }}(\theta, a)=\mathcal{E}_{\text {core }}^{\text {partial }}\left(\theta, r_{c}\right)+\frac{1}{2 L_{\|}}\left(E_{e l}^{s}\left(\theta, r_{c}\right)-E_{e l}^{n s}(\theta, a)\right) .
$$

Finally, for the different values of $a$, we determine the coefficients $c_{0}, c_{1}$ and $c_{2}$ of Eq. (11) by fitting $e_{\text {core }}(\theta, a)$ with Eq. (11). The values of $e_{\text {core }}(\theta, a)$ obtained from Eq. (32) and the fitted functions are represented in Fig. 6.a for different values of $a$.
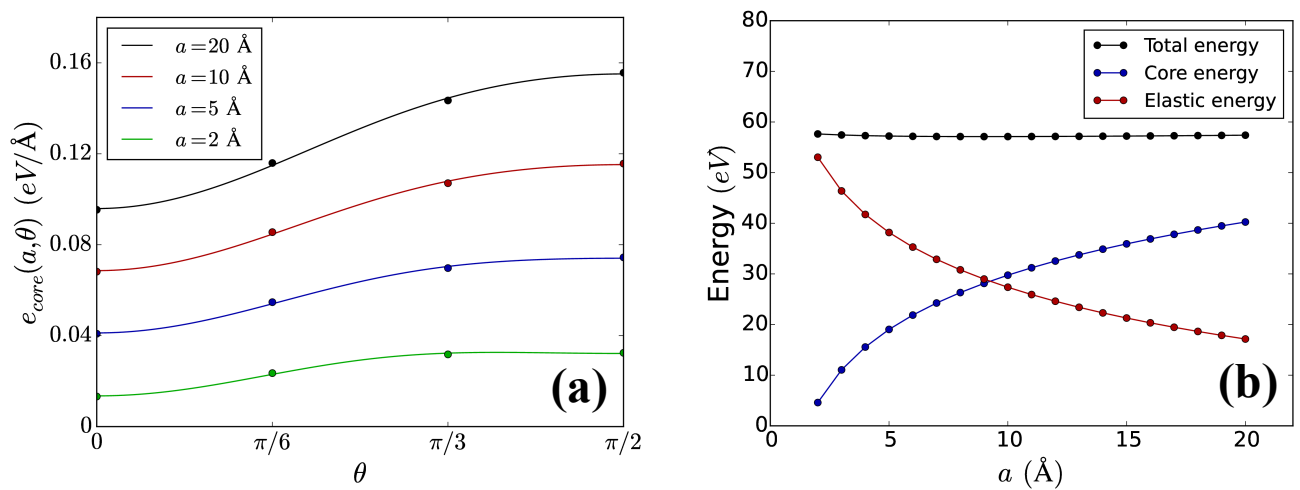

Figure 6: (a) Core energy obtained for different values of the parameter $a$. The dots represent the data obtained from the procedure explained in the text and the continuous lines are the fits performed against Eq. (11). (b) Core and elastic contributions for different regularization parameters $a$ of a circular loop of radius $R_{\text {loop }}=50 \AA$ in a periodic cubic system of size $L=200 \AA$.

To check the validity of this core energies parametrization, we considered a circular dislocation loop of radius $R_{\text {loop }}=50 \AA$ in a periodic cubic system of size $L=200 \AA$ and computed both the elastic and core energies for different values of $a$. The results are displayed in Fig. 6.b and show that for increasing values of $a$, the elastic energy of the system decreases (as expected) and the core energy compensates for this drop such that the total energy varies by less than $2 \%$ in the range of $a$ values explored here.

\subsection{Generalized stacking fault}

The generalized stacking fault (GSF) energy $e_{g s f}(\zeta)$ introduced in section 2.3 is classically obtained [57] by shifting two half crystals along a $\{111\}$ plane in the $[11 \overline{2}]$ direction and relaxing the atomic positions in the [111] direction only. Performing these relaxations for different values of $\zeta$ yields the stacking fault energy represented with blue dots in Fig. 7.b. 
As shown in Ref. [14], this GSF can vary significantly with the applied shear stress and this effect has to be taken into account in the DD simulation to reproduce the appropriate atomistic activation energy. For simplicity reasons, we considered in the present work only the effect of a shear stress $\sigma^{a}$ in the $[11 \overline{2}]$ direction, as depicted in Fig. 7.a. The resulting curves are shown as full lines in Fig. 7.b. We note that the position of the GSF local minimum $b_{p}\left(\sigma^{a}\right)$ increases slightly with increasing stress, while the stacking fault energy $U_{s f}\left(\sigma^{a}\right)$ (i.e. the value of the GSF at $\left.b_{p}\left(\sigma^{a}\right)\right)$ decreases.
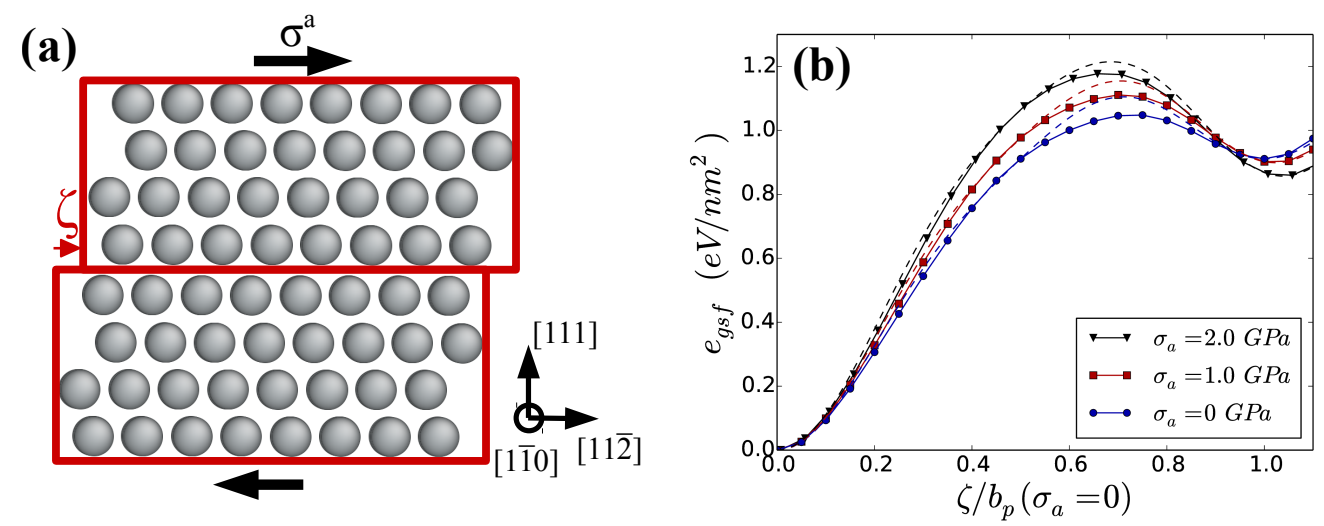

Figure 7: (a) Sketch showing the method to compute the GSF under an applied shear stress. (b) GSF energy in the $[11 \overline{2}]$ direction for different applied stresses and corresponding fits using Eq. (33).

A way to incorporate the GSF energy in the model is to represent the atomistic data by a cosine series of the form:

$$
e_{g s f}\left(\zeta, \sigma^{a}\right)=a_{1}\left(1-\cos \left(\frac{\pi \zeta}{b_{p}}\right)\right)+a_{2}\left(1-\cos \left(\frac{2 \pi \zeta}{b_{p}}\right)\right)+a_{3}\left(1-\cos \left(\frac{4 \pi \zeta}{b_{p}}\right)\right)
$$

This functional form accounts for the periodicity of the crystal lattice and forces $\zeta=0$ and $\zeta=b_{p}$ to be local minima of $e_{g s f}(\zeta)$. Moreover, such analytical forms are much easier to handle computationally than tabulated data. The parameters $a_{i}$ are determined with the following procedure: we first extract different physical values from the atomistic GSF: the amplitude of the Burgers vector $b_{p}$, the shear elastic modulus $C$ in the direction of the Shockley Burgers vector around $\zeta=0$, the interplanar distance $h$, the value of the stacking fault $U_{s f}$ and the value of the GSF for $\zeta=b_{p} / 2$ noted $U_{1 / 2}$. We then impose the following conditions on the function $e_{g s f}(\zeta)$ :

$$
\begin{aligned}
e_{g s f}\left(b_{p}\right) & =U_{s f} \\
e_{g s f}\left(\frac{b_{p}}{2}\right) & =U_{1 / 2} . \\
\left.\frac{\partial^{2} e_{g s f}}{\partial^{2} \zeta}\right|_{\zeta=0} & =\frac{C}{h}
\end{aligned}
$$


With such additional conditions, the values of the coefficients $a_{i}$ in Eq. (33) become uniquely fixed. The resulting solutions calculated for different applied stresses are plotted with dashed lines in Fig. 7.b. They reproduce accurately the atomistic curves close to the minima $\zeta \simeq 0, b_{p}$ but lack precision around the GSF maximum. The latter problem could be minimized by expanding the cosine series of Eq. (33) but this is unnecessary in the case of partial nucleation. Indeed, saddle configurations of the atomistic results reported in section 1 are found for wellformed loops for which the disregistery is close to $\zeta=b_{p}$. Therefore, the value of the unstable stacking energy does not influence the activation energy of the nucleation process for the stress levels investigated in this work.

\section{Homogeneous nucleation of partial dislocations}

We now turn to the study of homogeneous partial dislocation nucleation by incorporating the NEB algorithm detailed in section 1 in the variational dislocation dynamics model of section 2 carefully parametrized on atomistic simulations. Compared to atomistic simulations, the DoFs are now the positions of the nodes along the dislocation lines, with nodal forces computed as derivatives of the system energy including elastic, core and stacking fault contributions. In this paragraph, we wish to compare the results of VDD simulations to atomistic calculations for the simple case of homogeneous nucleation under shear stress but we point out that the present model is applicable to more complex cases such as heterogeneous nucleation discussed in section 5 .

As in the atomistic simulations performed in section 1, the last replica representing the largest loop is treated with the Free-End NEB (FENEB) method [5] and evolves at constant energy while being subjected to the spring force from its neighboring replica. In addition, the VDD model can not deal with loop radii $R_{\text {loop }} \lesssim a$. For this reason, the first NEB replica also contains a loop of finite size, which is evolved at constant energy using the FENEB method. Thus, the first and final configurations, chosen on both sides of the energy barrier, evolve during the NEB minimization, but are not allowed to reach a minimum because they are constrained to remain at a constant energy.

We note that, in contrast with most dislocation dynamics models, we rely here on a fixed number of DoFs in the different replicas of the NEB path. The number of nodes along the dislocation line is thus fixed along the NEB path. With other dislocation mechanisms (e.g. bowing out of a dislocation), the dislocation shape and topology may evolve significantly along the NEB path, and fixing the number of DoFs might become an obstacle to obtain reliable results. To treat such cases, the present NEB approach could be extended to incorporate a varying number of DoFs along the path, for example by splitting nodes in regions of high curvature and linking one DoF in a replica to two or more DoFs in the neighboring replica.

To avoid global translations of the replicas, the forces are corrected to remove translational modes. The replica of maximum energy is treated with the climbing image method [21] to ensure its convergence to the saddle configuration. The quenched dynamics relaxation algorithm is run until all nodal forces are below a threshold. The latter, noted $f_{t h}$, is physically chosen by computing the minimum segment length, $L_{m i n}$, in all replicas and 

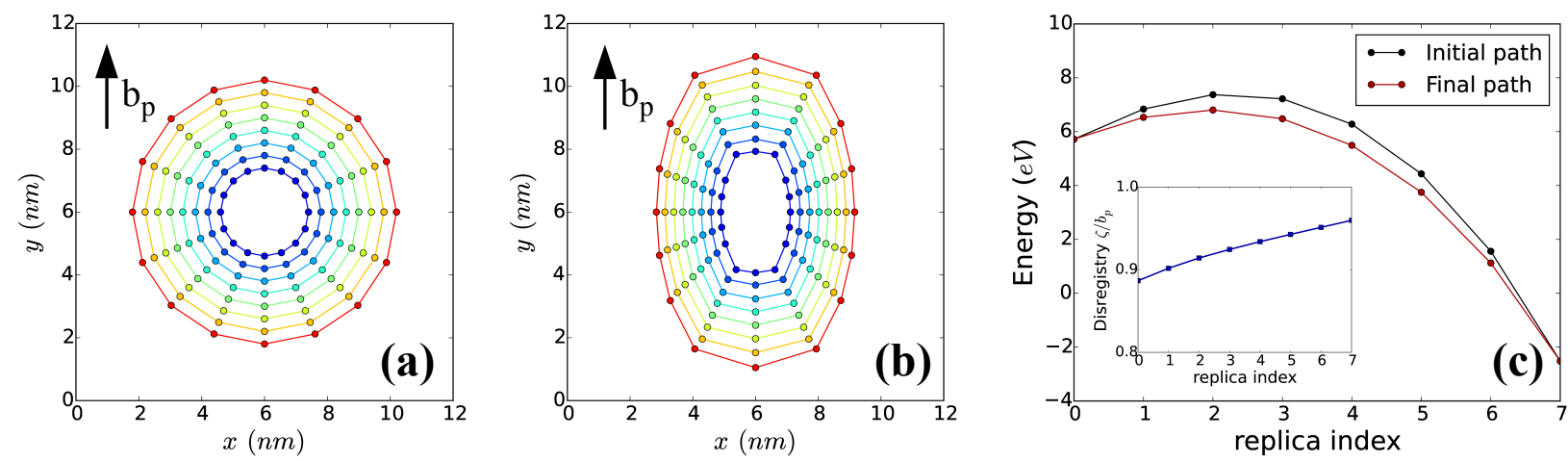

Figure 8: NEB calculations performed at $\sigma^{a}=1.6 \mathrm{GPa}, a=4 \AA$ and $N_{\text {rep }}=8$ replicas of a loop with $N_{\text {nodes }}=16$ nodes: (a) initial path, (b) final relaxed path, (c) energy of the replicas in the initial and final paths and the disregistry along the final path in the inset.

setting $f_{t h}=\sigma_{t h} b L_{m i n}$, where $\sigma_{t h}=20 \mathrm{MPa}$ is of the same order of magnitude as the Peierls stress in aluminum [58].

We first consider the case of elastic isotropy. The initial path is composed of 8 circular loops of increasing radii. The system size varies from $L_{x}=12 \mathrm{~nm}$ to $L_{x}=30 \mathrm{~nm}$, depending on the applied stress and on the size of the critical loop. Elastic fields are computed on a regular cubic grid of $256^{3}$ grid points. For each system size, the regularization parameter $a$ is taken such that $3 d x<a<2 R_{\text {min }}$, such that even the smallest replica of radius $R_{\min }$ is described accurately.

As an example, Fig. 8 displays the initial and final paths obtained at a stress $\sigma^{a}=1.6 \mathrm{GPa}$ with $a=4 \AA$, as well as the energy of the initial and relaxed paths (Fig. 8.c). As expected, the minimum energy path is a series of loops elongated in the direction of the Burgers vector, because the elastic line energy is minimum in the screw orientation. One can notice that the FENEB treatment for the first and last replicas allows a modification of the loop shapes at constant energy. We also note that after minimization, the disregistery of the saddle configuration (replica \#2) is $\zeta=0.914 b_{p}$, significantly lower that the full Burgers vector $b_{p}$, revealing the importance of including the generalized stacking fault energy and the minimization of Eq. (21) in the numerical model.

We performed similar simulations at different stress levels and compare in Fig. 9 the resulting activation energies with atomistic calculations. We find an excellent agreement between both approaches in the stress range where both are applicable. The agreement is all the more satisfactory that there is no fitting parameter: the parameters of the VDD model were carefully chosen based on atomistic data but none were fitted to reproduce this specific result. In addition, it is noticeable that the methodology applied to parametrize the dislocation core energy (see section 3.1) involves straight dislocations but is able to reproduce atomistic data involving curved dislocations. Lastly, it must be noted that, as expected, the present model allows to explore smaller applied stresses than atomistic simulations: at $1200 \mathrm{MPa}$, the saddle configuration is found for a $20 \mathrm{~nm}$ diameter loop, at the computational 
limit of atomistic NEB calculations.

Looking carefully at Fig. 9, we notice that the activation energies predicted by the VDD model underestimate slightly the atomistic results. This can be attributed to small differences between the VDD and atomistic calculations. First, as shown in Fig. 7.a, in the atomistic calculations the elastic shear constant (proportional to the second derivative of the GSF at $\zeta=0$ ) increases with stresses, making the material stiffer at high stresses. This effect was not incorporated in the VDD model where the elastic constants were assumed independent of the applied stress. Secondly, slightly different boundary conditions may also have a small influence: while the VDD simulations are tri-periodic, the atomistic simulations include free surfaces in $\pm z^{3}$. We also note that the VDD simulations can not explore dislocation nucleation at stresses higher than $1.9 \mathrm{GPa}$ because at these high stress levels, when solving Eq. (21) for the first image of the NEB path, the optimum value of $\zeta$ is found close to 0 instead of $b_{p}$ and the minimization procedure fails to find the minimum energy path. However, this region of high-stresses is not the target of the present method since atomistic calculations are then applicable. We note that this limitation is expected to be less severe in the case of other FCC metals where the stacking fault energy is lower than in aluminum.

A noticeable advantage of the VDD model compared to atomistic calculations is its efficiency: at $\sigma^{a}=1.6 \mathrm{GPa}$, the VDD calculation takes about 1 day on a single processor while atomistically, it takes about 1 week on 8 processors (i.e. a $\times 56$ speed up). This is attributed to the number of DoFs that is considerably reduced from atomistic (10 replicas with 133056 atoms, i.e. 3991680 DoFs in total) to VDD calculations (8 replicas with 16 nodes, i.e. 256 DoFs in total). In the appendix, we present a more detailed analysis of the numerical efficiency of the VDD approach compared to atomistic simulations. We show in particular that, in constrast with atomistic simulations, our VDD model enables to investigate thermally activated processes occuring on large length-scales at no additional computational cost.

As explained in section 3, the regularization parameter $a$ is considered here as a numerical parameter. Therefore, the results should only depend marginally on its value. This is checked in Fig. 10.a where the activation energy is shown as a function of $a$ for different stresses. We note the weak effect of this parameter on the results, varying within a $10 \%$ range despite the large variation of $a$.

Another numerical parameter in these calculations is the number of nodes along the loops. Fig. 10.b shows that the activation energy does not vary significantly when increasing the number of nodes, confirming that 16 nodes are enough to properly describe the loops.

An advantage of solving the elastic problem numerically using an eigenstrain formalism is that elastic anisotropy can be incorporated straightforwardly. To investigate its influence, we performed anisotropic simulations starting from the minimum energy paths found with isotropic elasticity. As shown in Fig. 11.a, the energy changes very slightly between the anisotropic and isotropic case. This result must be attributed to the weak anisotropic factor of aluminum.

\footnotetext{
${ }^{3}$ Using tri-periodic atomistic cells is possible but would require to apply the NEB method in cells of different shapes along the path, which is technically difficult and requires additional computational time.
} 


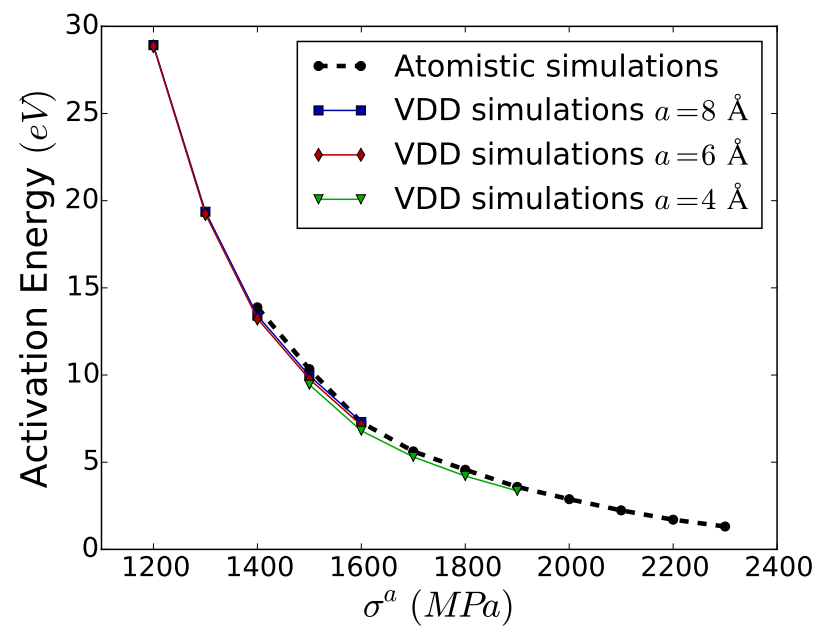

Figure 9: Activation energy for loop nucleation as a function of applied stress from atomistic (dash line) and VDD calculations. Different values of the regularization parameter $a$ were used, as indicated in the legend.
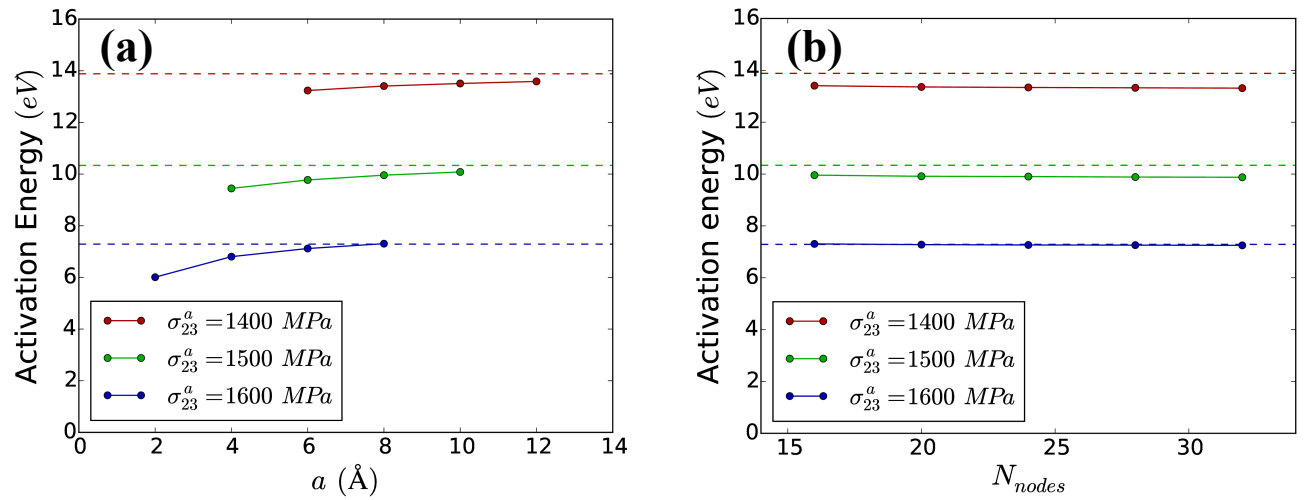

Figure 10: Variation of the activation energy as a function of the regularization parameter (a) and the number of nodes (b). The activation energies found in atomistic simulations are shown as dashed lines. 

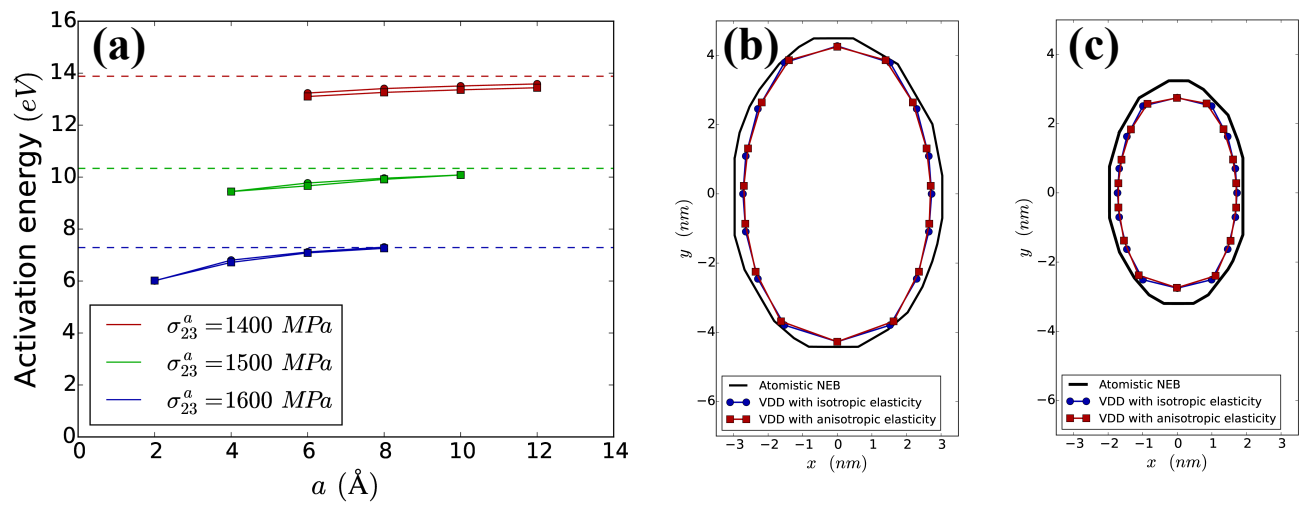

Figure 11: (a) Effect of elastic anisotropy on the activation energy for various values $\sigma_{a}$ and $a$. Circular (resp. square) data points are obtained from isotropic (resp. anisotropic) elasticity. (b-c) Comparison of the loop shapes for (b) $\sigma_{a}=1.4 \mathrm{GPa}$ and (c) $\sigma_{a}=1.6 \mathrm{GPa}$ between atomistic calculations (black lines) and VDD calculations with isotropic (blue dots) and anisotropic (red squares) elasticity.

Fig. 11.b-c shows the loop shapes obtained from atomistic and VDD calculations with both isotropic and anisotropic elasticity. The atomistic loop shape is obtained by extracting the defected atoms and plotting their convex envelope. Once again, we highlight the very good agreement between the VDD and atomistic calculations. The slight differences between the two methods are attributed to assumptions of the VDD model. In particular, the increase of the elastic moduli with the applied stresses (as shown in Fig. 7) is not incorporated in the VDD approach, and the softer elastic constants in the VDD simulations lead to smaller critical nucleii compared to the atomistic calculations. Another source of discrepancy might come from the Peierls potential acting on partial dislocations. While this effect is neglected in the VDD simulations where the energy is translation-invariant, the lattice friction might have an influence on the atomistic results. Moreover, variation of the Peierls potential with dislocation orientation may also influence the loop shape by "trapping" some dislocation character more than others.

Also, comparing closely the loop shapes obtained with isotropic and anisotropic elasticity, we notice that the latter is slightly asymmetric (the $x$-axis is no longer an axis of symmetry of the loop). This asymmetry is a weak effect in the case of Al but remains a physical effect of elastic anisotropy that has already been pointed out in dislocation dynamics simulations in iron [59].

\section{Heterogeneous nucleation around precipitates.}

An advantage of the present NEB calculations in a DD simulation code making use of the eigenstrain theory is that the effect of heterogeneous stresses coming from a misfitting particle or other types of defects is straightforward to incorporate. In particular, the present model can be applied to investigate the nucleation of dislocations around misfitting precipitates, a process that has long been proposed to lead to coherency loss of precipitates with the 
surrounding matrix $[60,61,62,40]$. As examples, we can mention precipitation hardened aluminum alloys such as $\mathrm{Al}-\mathrm{Sc}$ and $\mathrm{Al}-\mathrm{Zr}$ alloys, where misfitting $\mathrm{Al}_{3} \mathrm{Sc}$ and $\mathrm{Al}_{3} \mathrm{Zr}$ precipitates loose their coherency with the matrix upon thermal treatment [63, 64]. This property is known to affect both the coarsening behavior of the particles and the mechanical properties of the resulting alloy $[65,66]$.

In this section, we propose to use our new model to investigate the nucleation of a dislocation loop in the vicinity of a misfitting particle and compute the corresponding energy barrier. This work allows to go beyond the estimate proposed by Ashby and Johnson [61] by letting the NEB algorithm find the shape of the loop along the minimum energy path.

We note that such a problem could be studied using the quasi-continuum approach $[67,68]$ : the large dislocation-free part of the system can be modeled elastically with a finite-element solver while an atomistic small volume is incorporated close to the precipitate interface to investigate the nucleation process, provided that the hybrid scheme remains variational. We mention also the approach of Kim et al. [69] that incorporates a temperature accelerated method in a quasi-continuum approach, which could in principle be used to investigate dislocation nucleation events in the vicinity of a precipitate.

For simplicity reasons, we consider precipitates with a misfit $\varepsilon_{0}=3 \%$ and isotropic elastic constants identical to the aluminum matrix. We point out however than the elastic solver used for the VDD calculations could be extended to treat heterogeneous elastic moduli by using an iterative method in Fourier space [70, 71, 72]. To avoid sharp variation of the stresses around the precipitate interface, we introduce a finite interface width in the range $w_{i}=0.5-2 \mathrm{~nm}$, depending on the grid spacing $d x$ and satisfying $R \gg w_{i} \simeq 2 d x$. The coherency loss mechanism can either involve the nucleation of a partial dislocation loop as discussed above or the nucleation of a perfect dislocation. The model was therefore adjusted to treat the nucleation of perfect dislocations: following the procedure described in section 3.1, we computed the core energy of perfect dislocations and parametrized the model accordingly.

We first consider a precipitate of radius $R_{p p t}=66 \mathrm{~nm}$ and introduce a small partial dislocation loop of circular shape at a height $z=46.67 \mathrm{~nm}$ from the center of the precipitate (i.e. at an angle $\psi=45^{\circ}$ with respect to the equatorial plane of the inclusion). A notable difference with homogeneous nucleation is that because the stress decreases away from the precipitate, the dislocation loop reaches an equilibrium configuration after nucleation. The system is then relaxed using quenched dynamics until the nodal forces reach the threshold value $f_{t h}$. The resulting stable configuration is shown in Fig. 12.a. Starting from this equilibrium configuration, we then build a path formed of smaller concentric loops. The free-end NEB is applied to the smaller loop while the larger one is maintained fixed. The minimization procedure converges towards the minimum energy path represented in Fig. 12.b, with the energy of the initial and final paths shown in Fig. 12.c. We checked that the same solution was found starting from different initial paths, therefore showing that the converged path does not depend on the initial configuration. We also point out that the saddle configuration (replica \#2 represented with a thick black line in Fig. 12.b) does not fit closely the precipitate interface but takes a rather circular shape ahead of the interface. 

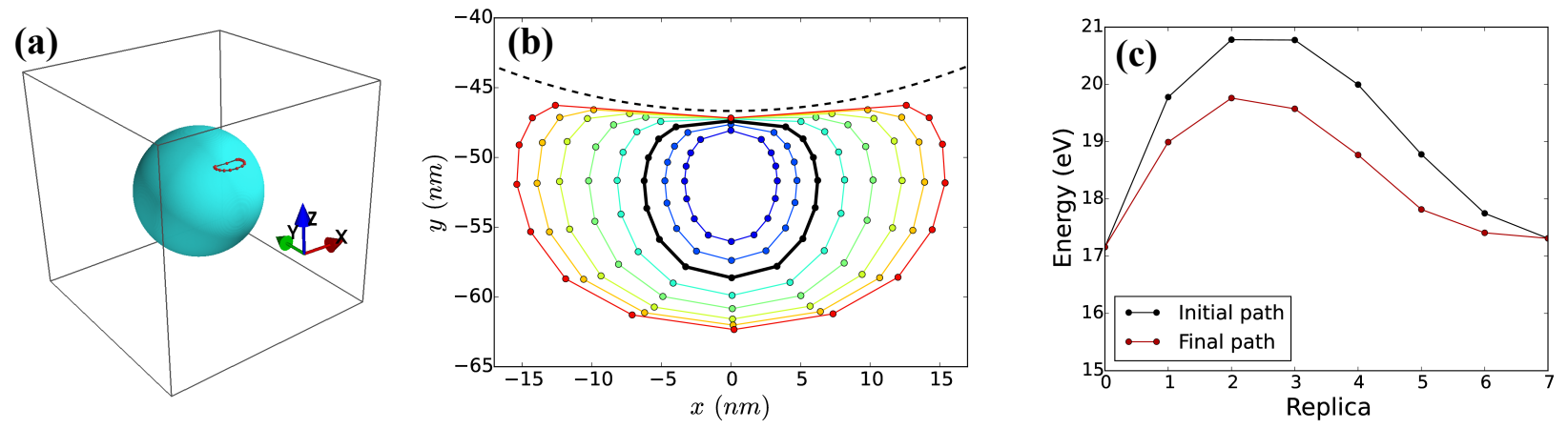

Figure 12: (a) Equilibrium configuration of a partial Shockley dislocation around a spherical inclusion of radius $R=66 \mathrm{~nm}$ and eigenstrain $\varepsilon_{0}=3 \%$. (b) Relaxed NEB path for the nucleation of a partial dislocation loop. The precipitate interface is shown with a dashed line. (c) Energy of the replicas in the initial and final paths (the $0 \mathrm{eV}$ state corresponds to the dislocation-free system).

We now turn to the activation energy of the nucleation process depending on the loop position with respect to the precipitate interface. Previous studies [60, 61, 62] have assumed nucleation to occur at an angle $\psi=45^{\circ}$, where the shear stress at the interface is maximum. However, the saddle configuration such as the one shown in Fig. 12.b has a finite size and the entire stress profile around the precipitate affects the activation energy rather than the maximum stress alone. Therefore, we investigated the nucleation events at different angles $\psi$ defined in Fig. 13.a. In the case of partial nucleation, Fig. 13.b and c show that the minimum activation energy is found for $\psi \simeq 50^{\circ}$ and $\psi \simeq 55^{\circ}$ for partial and perfect dislocations respectively. This can be explained by examining the stress field in front of the precipitate, or more precisely the following integral

$$
\begin{aligned}
W_{p k} & =\int_{R_{p p t} \cos \psi}^{R_{p p t}(1+\cos \psi)} \int_{-R_{p p t} / 2}^{+R_{p p t} / 2} b \sigma_{23}^{p p t}(x, y, z) d x d y \\
& =-\frac{2(1+\nu) \mu \varepsilon_{0} z b R_{p p t}^{3}}{(1-\nu)} \int_{R_{p p t} \cos \psi}^{R_{p p t}(1+\cos \psi)} \int_{-R_{p p t} / 2}^{+R_{p p t} / 2} \frac{y d x d y}{\left(x^{2}+y^{2}+z^{2}\right)^{5 / 2}},
\end{aligned}
$$

representing the work of the Peach-Koehler force coming from the precipitate stress field $\sigma_{23}^{p p t}$ when considering the expansion of a square shaped loop depicted in Fig. 13.d. Assuming this simple square for the nucleated dislocation yields an analytical expression for this integral and the difference $W_{p k}(\psi)-W_{p k}\left(45^{\circ}\right)$ is represented as a function of the angle $\psi$ in Fig. 13.d for different precipitate radii. It shows that Eq. (37) is minimum for $\psi \simeq 71^{\circ}$ for the different precipitate radii, clarifying why the activation energies reported in Fig. 13.b and c present minima for $\psi>45^{\circ}$. We note that, in addition to the work of the Peach-Koehler force, the calculations show that other energy contributions (self-energy of the loop, stacking fault energy and core energy) have a significant effect on the activation energy.

As shown in Fig. 13.b and 13.c, the activation energy decreases with increasing precipitate radius $R_{\text {ppt }}$. This is expected since the characteristic length scale of the stress field around 

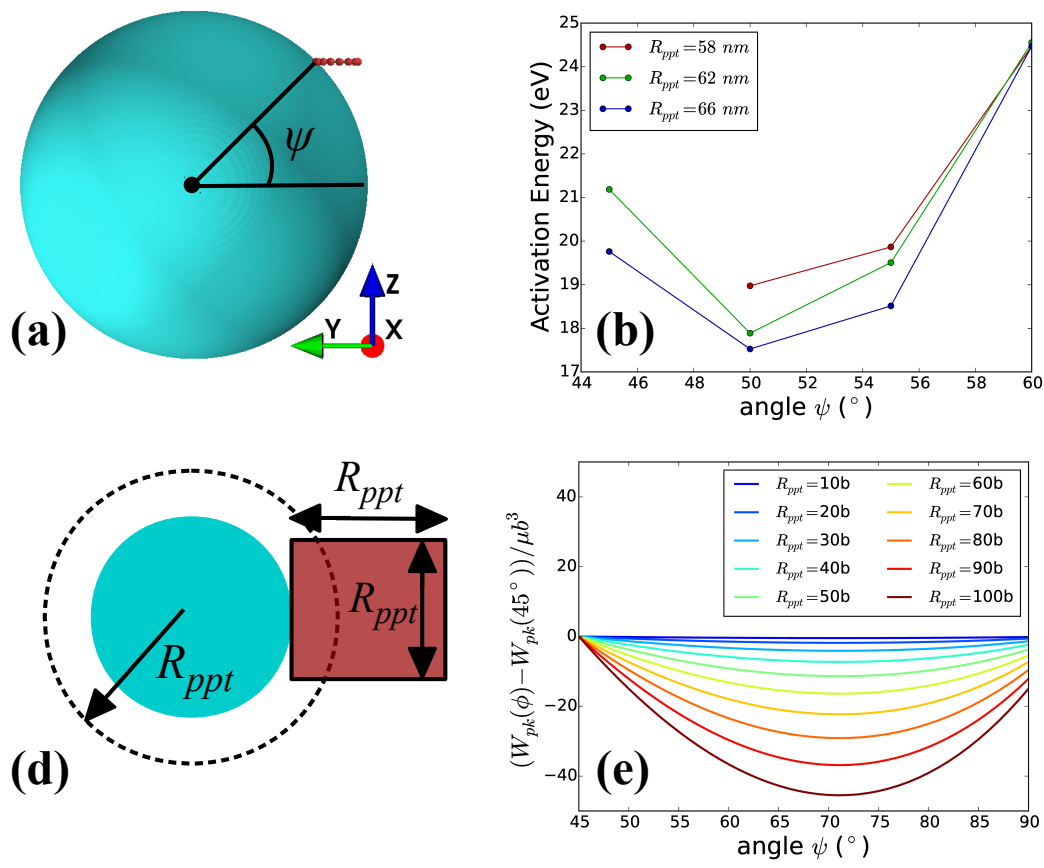
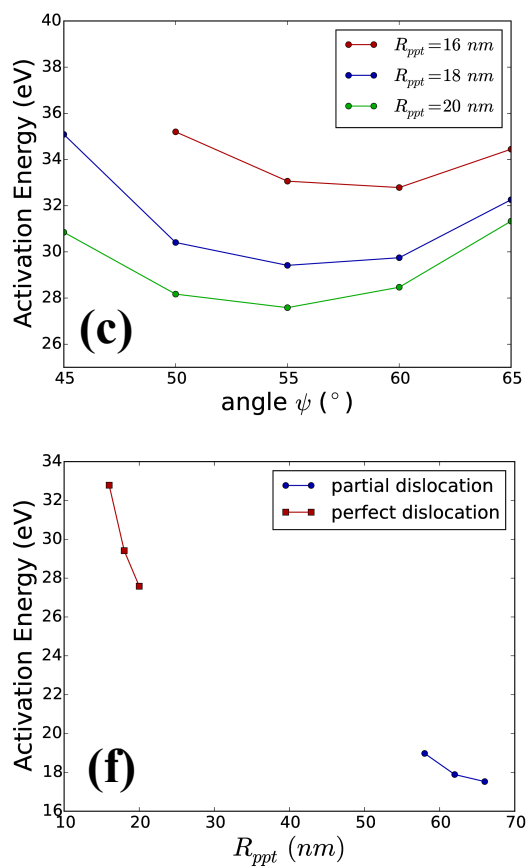

Figure 13: (a) Snapshot of Fig. 12.a seen from the side and showing the definition of the angle $\psi$. (b) Activation energy function of the angle $\psi$ for different precipitate sizes in the case of partial (b) and perfect (c) dislocations. (d) Sketch of the precipitate seen from above and the simplified geometry of the loop (shown in pale red) considered in Eq. (37). (e) Difference $W_{p k}(\psi)-W_{p k}\left(45^{\circ}\right)$ defined in Eq. (37) as a function of $\psi$ for different loop radii. (f) Minimum activation energy found in the case of partial and perfect dislocations function of the precipitate radius. 
the precipitate scales with the precipitate radius. Therefore, the activation energy is lowered due to an increase of the work of the Peach-Koehler force.

The minimum activation energy is shown in Fig. 13.f as a function of the precipitate radius for both partial and perfect dislocations. We note that, in both cases, there is a threshold precipitate radius $R_{t h}$, respectively $R_{t h}=15 \mathrm{~nm}$ for perfect and $R_{t h}=58 \mathrm{~nm}$ for partial dislocations, below which any dislocation is unstable in the vicinity of the precipitate: for any loop sizes and positions, the self energy of the loop is larger than the work of the Peach-Koehler force and the loop collapses. The value of this threshold radius is much higher in the case of partials due to the contribution of the stacking fault energy. Moreover, we did not investigate the nucleation process for every precipitate radii above $R_{t h}$ : for values $R_{p p t} \gg R_{t h}$, the saddle configuration becomes very small compared to the size of the simulation and the calculation necessitates a small value of the regularization parameter $a$ to describe accurately this saddle configuration, making these simulations difficult to achieve computationally. We therefore restricted ourselves to values of $R_{p p t}$ just above $R_{t h}$ as shown in Fig. 13.f that can be investigated with a $256^{3}$ grid. This prevents us to conclude definitely on the coherency loss mechanisms (either through partial or perfect nucleation) but we note that the nucleation of partial is much less likely since partial loops are stable only for large precipitate radii.

Finally, the activation energies reported in Fig. 13.b and 13.c are unreasonably high for both the partial and perfect cases. These high-energy barriers can be discussed in light of a recent study focusing on dislocation nucleation on voids [73] using the finite-temperature string method. The authors also obtain very high nucleation energy barriers for reasonable applied stresses and conclude that nucleation on voids is unlikely to occur under ordinary experimental conditions. However, a limitation of both studies lies in the interface, which remains idealized. Indeed, the presence of defects (vacancy, ledges, etc.) at the interface and fluctuations of the interface shape may lower the activation energy of the nucleation process considerably, facilitating the formation of interface dislocations, as mentioned by Ashby and Johnson [61]. The influence of these defects are however difficult to take into account in our model where the complexity of the crystal structure has been simplified to promote computational efficiency.

\section{Conclusion}

In this work, we have incorporated the nudged elastic band method in a dislocation dynamics formalism. A nodal representation of dislocations has been employed and a careful attention has been paid to the variational character of the model. The model parameters such as the dislocation core energy and the generalized stacking fault energy were obtained from atomistic simulations using an interatomic potential for aluminum. We applied our calibrated model to textbook problems of thermally activated dislocation nucleation. A remarkable agreement is found between the results of our model and atomistic calculations for the homogeneous nucleation of partials. We have also investigated the influence of elastic anisotropy, having a weak effect in the case of aluminum. Then, we have applied our model to investigate dislocation nucleation around a spherical precipitate; our main result being that 
the minimum activation energy is found above the plane oriented at $\psi=45^{\circ}$ like assumed in previous studies.

The main advantage of our continuous approach compared to atomistic calculations relies on its computational efficiency. As illustrated in the Appendix below, the present model can be used to investigate dislocation-mediated mechanisms at large length-scales for no additional computational cost thanks to our parametrization procedure, which allows to use fixed grid sizes for increasing simulated volumes. This is in contrast with atomistic NEB, whose convergence time increases drastically with the simulated volume. Moreover, while atomistic calculations are usually performed at $0 \mathrm{~K}$, the parameters of the VDD model can be chosen to account for finite temperature material properties, enabling to obtain the temperature-dependent term of the activation energy, known to lower significantly the energy barrier for dislocation nucleation [15].

We also note that the NEB approach presented here is directly transferable to standard nodal dislocation dynamics models [33, 74], where the stresses and energy are computed based on analytical equations valid in an infinite medium. However, a prerequisite for the application of the NEB method must be that the formalism is variational and the forces are derivatives of the energy. Such a model would be less general than the present approach because the elastic influence of interfaces (grain boundaries, precipitate interfaces, etc.) and elastic anisotropy would be more difficult to incorporate, but the computation of forces and energies would be significantly faster, allowing to investigate thermally activated events in large dislocation microstructures.

The implementation of the nudged elastic band method in DD simulations opens the way to the study of many other thermally activated processes in microstructures of dislocations out of the reach of atomistic simulations. As a matter of examples, we mention dislocation bowing and passing features in persistent slip band [75] or the motion of low angle grain boundary, encountered during recrystallization [76]. Moreover, our variational approach is a framework of choice to transfer other numerical methods from atomistic systems to the study of dislocation ensembles: the activation-relaxation technique $[9,10,11]$ and accelerated dynamics methods $[77,78,79]$ could allow to investigate thermally activated events in complex systems when the final state is unknown. The variational aspect of the model also makes possible a self-consistent coupling between dislocation dynamics and other physical phenomena such as phase transformations and fracture, thus enabling to investigate the in-

teractions between dislocations, precipitate evolution and crack propagation in a quantitative self-consistent way.

\section{Acknowledgements}

We would like to thank Christophe Denoual and Emmanuel Clouet for their interest in the project and their helpful comments. We acknowledge the support of the Agence Nationale de la Recherche through grant ANR-13-MERA-0001-02. 

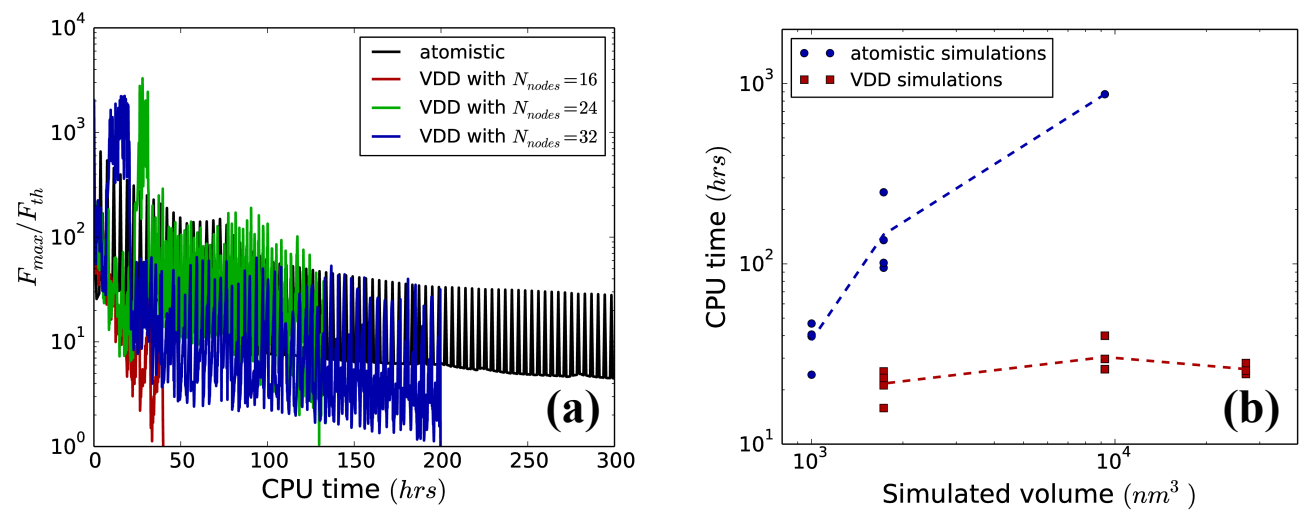

Figure 14: (a) Comparison of the convergence behavior of VDD simulations for different numbers of DoFs and corresponding atomistic calculations for the homogeneous nucleation event at $\sigma^{a}=1500 \mathrm{MPa}$. (b) Loglog plot of the convergence time for VDD and atomistic calculations as a function of the simulated volume. The average behavior is shown with a dashed line

\section{Appendix: computational efficiency}

One of the advantages of the VDD model is the reduction of the number of DoFs compared to atomistic calculations, where the position of each atom has to be tracked down. On the other hand, computing the elastic equilibrium with our spectral method comes at a rather elevated computational cost - especially with the fine grid used to describe the dislocation core - raising the question of the computational advantage of the VDD method compared to atomistic calculations.

In both VDD and atomistic calculations, the nudged elastic band method stops when the maximum component of the force $F_{\max }$ acting on the system becomes lower than a given threshold denoted $F_{t h}$. Fig. 14.a displays the ratio $F_{\max } / F_{t h}$ as a function of simulation time (measured as CPU hours) for different VDD simulations and for an atomistic simulation. We first point out the jagged behavior of the curves, attributed to the frequent updates of the NEB tangent during the minimization procedure. Second, we note that the computational time associated to the VDD simulations increases significantly with the number of nodes along the line. This behavior is characteristic of the NEB method for which the convergence time strongly increases with the number of DoFs. However, this convergence time also strongly depends on the initial path, on the convergence criteria, on the algorithm used for the minimization (see [80]) and on the problem at hand. A reliable scaling of the NEB is therefore difficult to obtain. Fig. 14 also shows that in the case of homogeneous nucleation, the convergence behavior of the atomistic simulation is much slower than VDD simulations.

To compare in more details the simulation times of VDD and atomistic calculations, we show in Fig. 14.b the CPU time as a function of the simulated volume. As expected, the convergence time of atomistic simulations increases rapidly with the volume, due to the increase of the number of DoFs in the system. In contrast, the VDD simulation time does not increase significantly because we keep the same number of DoF and the same $256^{3}$ underlying grid to 
solve the elastic problem. This is possible thanks to the parametrization procedure detailed in section 3.1, which considers the regularization parameter $a$ as a numerical parameter, which can be chosen freely and does not influence the results significantly (see Fig. 10.a). Therefore, when going to larger volumes, an increasing parameter $a$ can be chosen, such that the dislocation core remains well described on our $256^{3}$ grid.

\section{References}

[1] D. Caillard, J.-L. Martin, Thermally activated mechanisms in crystal plasticity, Elsevier, 2003.

[2] L. Kubin, Dislocations, mesoscale simulations and plastic flow, Oxford University Press, 2013.

[3] M. Kassner, Fundamentals of creep in metals and alloys, Butterworth-Heinemann, 2015.

[4] X. Li, Y. Wei, L. Lu, K. Lu, H. Gao, Dislocation nucleation governed softening and maximum strength in nano-twinned metals, Nature 464 (7290) (2010) 877-880. doi:10.1038/nature08929.

[5] T. Zhu, J. Li, A. Samanta, H. Kim, S. Suresh, Interfacial plasticity governs strain rate sensitivity and ductility in nanostructured metals, Proceedings of the National Academy of Sciences 104 (9) (2007) 3031-3036. doi:10.1073/pnas.0611097104.

[6] G. Mills, H. Jónsson, Quantum and thermal effects in $\mathrm{H}_{2}$ dissociative adsorption: evaluation of free energy barriers in multidimensional quantum systems, Physical Review Letters 72 (7) (1994) 1124. doi:10.1103/PhysRevLett.72.1124.

[7] G. Mills, H. Jónsson, G. Schenter, Reversible work transition state theory: application to dissociative adsorption of hydrogen, Surface Science 324 (2) (1995) 305-337. doi:10.1016/00396028(94)00731-4.

[8] H. Jónsson, G. Mills, K. Jacobsen, Nudged elastic band method for finding minimum energy paths of transitions, in: Classical and Quantum Dynamics in Condensed Phase Simulations - Proceedings of the International School of Physics, 1998, Ch. 16, pp. 385-404. doi:10.1142/9789812839664_0016.

[9] G. Barkema, N. Mousseau, Event-based relaxation of continuous disordered systems, Physical Review Letters 77 (21) (1996) 4358. doi:10.1103/PhysRevLett.77.4358.

[10] N. Mousseau, G. Barkema, Traveling through potential energy landscapes of disordered materials: The activation-relaxation technique, Physical Review E 57 (2) (1998) 2419. doi:10.1103/PhysRevE.57.2419.

[11] N. Mousseau, L. Béland, P. Brommer, J.-F. Joly, F. El-Mellouhi, E. Machado-Charry, M.C. Marinica, P. Pochet, The activation-relaxation technique: Art nouveau and kinetic art, Journal of Atomic, Molecular, and Optical Physics (2012) 925278.doi:10.1155/2012/925278.

[12] T. Zhu, J. Li, S. Yip, Atomistic study of dislocation loop emission from a crack tip, Physical Review Letters 93 (2) (2004) 025503. doi:10.1103/PhysRevLett.93.025503. 
[13] T. Zhu, J. Li, A. Samanta, A. Leach, K. Gall, Temperature and strain-rate dependence of surface dislocation nucleation, Physical Review Letters 100 (2) (2008) 025502. doi:10.1103/PhysRevLett.100.025502.

[14] S. Aubry, K. Kang, S. Ryu, W. Cai, Energy barrier for homogeneous dislocation nucleation: Comparing atomistic and continuum models, Scripta Materialia 64 (11) (2011) 1043-1046. doi:10.1016/j.scriptamat.2011.02.023.

[15] S. Ryu, K. Kang, W. Cai, Entropic effect on the rate of dislocation nucleation, Proceedings of the National Academy of Sciences 108 (13) (2011) 5174-5178. doi:10.1073/pnas.1017171108.

[16] A. Jennings, C. Weinberger, S.-W. Lee, Z. Aitken, L. Meza, J. Greer, Modeling dislocation nucleation strengths in pristine metallic nanowires under experimental conditions, Acta Materialia 61 (6) (2013) 2244-2259. doi:10.1016/j.actamat.2012.12.044.

[17] E. Maras, O. Trushin, A. Stukowski, T. Ala-Nissila, H. Jónsson, Global transition path search for dislocation formation in Ge on Si (001), Computer Physics Communications 205 (2016) 13-21. doi:10.1016/j.cpc.2016.04.001.

[18] Y. Mishin, D. Farkas, M. Mehl, D. Papaconstantopoulos, Interatomic potentials for monoatomic metals from experimental data and ab initio calculations, Physical Review B 59 (1999) 3393. doi:10.1103/PhysRevB.59.3393.

[19] S. Ohr, Displacement field around a shear dislocation loop, Philosophical Magazine 26 (6) (1972) 1307-1312. doi:10.1080/14786437208220344.

[20] G. Henkelman, H. Jónsson, Improved tangent estimate in the nudged elastic band method for finding minimum energy paths and saddle points, The Journal of Chemical Physics 113 (22) (2000) 9978-9985. doi:10.1063/1.1323224.

[21] G. Henkelman, B. Uberuaga, H. Jónsson, A climbing image nudged elastic band method for finding saddle points and minimum energy paths, The Journal of Chemical Physics 113 (22) (2000) 9901-9904. doi:10.1063/1.1329672.

[22] M. Gilbert, P. Schuck, B. Sadigh, J. Marian, Free energy generalization of the Peierls potential in iron, Physical Review Letters 111 (9) (2013) 095502. doi:10.1103/PhysRevLett.111.095502.

[23] C. Shen, J. Li, Y. Wang, Finding critical nucleus in solid-state transformations, Metallurgical and Materials Transactions A 39 (5) (2008) 976-983. doi:10.1007/s11661-007-9302-7.

[24] H. Kusumaatmaja, Surveying the free energy landscapes of continuum models: Application to soft matter systems, The Journal of Chemical Physics 142 (12) (2015) 124112. doi:10.1063/1.4916389.

[25] C. Semprebon, G. McHale, H. Kusumaatmaja, Contact angle and contact angle hysteresis in liquid infused and hemi-wicked surfaces (in press).

[26] G. Xu, A. Argon, M. Ortiz, Critical configurations for dislocation nucleation from crack tips, Philosophical Magazine A 75 (2) (1997) 341-367. doi:10.1080/01418619708205146. 
[27] G. Xu, C. Zhang, Analysis of dislocation nucleation from a crystal surface based on the peierlsnabarro dislocation model, Journal of the Mechanics and Physics of Solids 51 (8) (2003) 13711394. doi:10.1016/S0022-5096(03)00067-X.

[28] C. Sobie, L. Capolungo, D. McDowell, E. Martinez, Thermal activation of dislocations in large scale obstacle bypass, Journal of the Mechanics and Physics of Solids 105 (2017) 150-160. doi:10.1016/j.jmps.2017.05.003.

[29] C. Sobie, L. Capolungo, D. McDowell, E. Martinez, Scale transition using dislocation dynamics and the nudged elastic band method, Journal of the Mechanics and Physics of Solids 105 (2017) 161-178. doi:10.1016/j.jmps.2017.05.004.

[30] C. Sobie, L. Capolungo, D. McDowell, E. Martinez, Modal analysis of dislocation vibration and reaction attempt frequency, Acta Materialia.doi:10.1016/j.actamat.2017.02.005.

[31] C. Lemarchand, B. Devincre, L. P. Kubin, J. L. Chaboche, Coupled meso-macro simulations of plasticity: Validation tests, Vol. 538, MRS, Warrendale, Pennsylvania, 1999. doi:10.1557/PROC-538-63.

[32] O. Jamond, R. Gatti, A. Roos, B. Devincre, Consistent formulation for the discrete-continuous model: Improving complex dislocation dynamics simulations, International Journal of Plasticity 80 (2016) 19-37. doi:10.1016/j.ijplas.2015.12.011.

[33] B. Devincre, R. Madec, G. Monnet, S. Queyreau, R. Gatti, L. Kubin, Modeling crystal plasticity with dislocation dynamics simulations: The 'microMegas' code, Presses de l'Ecole des Mines de Paris, 2011.

[34] M. Verdier, M. Fivel, I. Groma, Mesoscopic scale simulation of dislocation dynamics in fcc metals: Principles and applications, Modelling and Simulation in Materials Science and Engineering 6 (6) (1998) 755. doi:10.1088/0965-0393/6/6/007.

[35] A. Arsenlis, W. Cai, M. Tang, M. Rhee, T. Oppelstrup, G. Hommes, T. Pierce, V. Bulatov, Enabling strain hardening simulations with dislocation dynamics, Modelling and Simulation in Materials Science and Engineering 15 (6) (2007) 553. doi:10.1088/0965-0393/18/4/045013.

[36] W. Cai, A. Arsenlis, C. Weinberger, V. Bulatov, A non-singular continuum theory of dislocations, Journal of the Mechanics and Physics of Solids 54 (3) (2006) 561-587. doi:10.1016/j.jmps.2005.09.005.

[37] J. Hirth, J. Lothe, Theory of Dislocations, McGraw-Hill, New York, 1968.

[38] G. Po, M. Lazar, D. Seif, N. Ghoniem, Singularity-free dislocation dynamics with strain gradient elasticity, Journal of the Mechanics and Physics of Solids 68 (2014) 161-178. doi:10.1016/j.jmps.2014.03.005.

[39] T. Mura, Micromechanics of defects in Solids, Kluwer Academic Publishers, Dordrecht, the Netherlands, 1993.

[40] P.-A. Geslin, B. Appolaire, A. Finel, Investigation of coherency loss by prismatic punching with a nonlinear elastic model, Acta Materialia 71 (2014) 80-88. doi:10.1016/j.actamat.2014.03.005. 
[41] P.-A. Geslin, Contribution à la modélisation champ de phase des dislocations., Ph.D. thesis, Université Pierre et Marie Curie - Paris VI (2013).

[42] P.-L. Valdenaire, Transport equations and dislocation density, Ph.D. thesis, Ecole Nationale Supérieure des Mines de Paris (2016).

[43] R. Scattergood, D. Bacon, The Orowan mechanism in anisotropic crystals, Philosophical Magazine 31 (1) (1975) 179-198. doi:10.1080/14786437508229295.

[44] R. Scattergood, D. Bacon, The strengthening effect of voids, Acta Metallurgica 30 (8) (1982) 1665-1677. doi:10.1016/0001-6160(82)90188-2.

[45] C. Kittel, Introduction to Solid State Physics, sixth Edition, John Wiley \& Sons, 1966.

[46] G. Simmons, H. Wang, et al., Single crystal elastic constants and calculated aggregate properties, MIT Press, 1971.

[47] K. Westmacott, R. Peck, A rationalization of secondary defect structures in aluminium-based alloys, Philosophical Magazine 23 (183) (1971) 611-622. doi:10.1080/14786437108216407.

[48] E. Clouet, Babel, http://emmanuel.clouet.free.fr/Programs/Babel/ (2010-2017).

[49] E. Bitzek, Atomistic simulation of dislocation motion and interaction with crack tips and voids, Ph.D. thesis, Universität Karlsruhe (2006).

[50] E. Clouet, Elastic energy of a straight dislocation and contribution from core tractions, Philosophical Magazine 89 (19) (2009) 1565-1584. doi:10.1080/14786430902976794.

[51] E. Clouet, L. Ventelon, F. Willaime, Dislocation core energies and core fields from first principles, Physical Review Letters 102 (2009) 055502. doi:10.1103/PhysRevLett.102.055502.

[52] E. Clouet, Dislocation core field. I. Modeling in anisotropic linear elasticity theory, Physical Review B 84 (22) (2011) 224111. doi:10.1103/PhysRevB.84.224111.

[53] J. Eshelby, W. Read, W. Shockley, Anisotropic elasticity with applications to dislocation theory, Acta Metallurgica 1 (3) (1953) 251-259. doi:10.1016/0001-6160(53)90099-6.

[54] A. Stroh, Dislocations and cracks in anisotropic elasticity, Philosophical Magazine 3 (30) (1958) 625-646. doi:10.1080/14786435808565804.

[55] W. Cai, V. Bulatov, J. Chang, J. Li, S. Yip, Periodic image effects in dislocation modelling, Philosophical Magazine 83 (5) (2003) 539-567. doi:10.1080/0141861021000051109.

[56] X. Shi, Etude par simulations de dynamique des dislocations des effets d'irradiation sur la ferrite à haute température, Ph.D. thesis, Université Pierre et Marie Curie - Paris VI (2014).

[57] V. Vitek, Dislocation cores and unconventional properties of plastic behavior, in: Handbook of Materials Modeling, Springer, 2005, pp. 2883-2896. doi:10.1007/978-1-4020-3286-8_174. 
[58] G. Lu, N. Kioussis, V. Bulatov, E. Kaxiras, Generalized-stacking-fault energy surface and dislocation properties of aluminum, Physical Review B 62 (5) (2000) 3099. doi:10.1103/PhysRevB.62.3099.

[59] J. Yin, D. M. Barnett, W. Cai, Efficient computation of forces on dislocation segments in anisotropic elasticity, Modelling and Simulation in Materials Science and Engineering 18 (4) (2010) 045013. doi:10.1088/0965-0393/18/4/045013.

[60] G. Weatherly, Loss of coherency of growing particles by the prismatic punching of dislocation loops, Philosophical Magazine 17 (148) (1968) 791-799. doi:10.1080/14786436808223030.

[61] M. Ashby, L. Johnson, On the generation of dislocations at misfitting particles in a ductile matrix, Philosophical Magazine 20 (167) (1969) 1009-1022. doi:10.1080/14786436908228069.

[62] L. Brown, G. Woolhouse, The loss of coherency of precipitates and the generation of dislocations, Philosophical Magazine 21 (170) (1970) 329-345. doi:10.1080/14786437008238420.

[63] K. Knipling, D. Dunand, D. Seidman, Precipitation evolution in Al-Zr and Al-Zr-Ti alloys during isothermal aging at $375-425^{\circ} \mathrm{C}$, Acta Materialia 56 (1) (2008) 114-127. doi:10.1016/j.actamat.2007.09.004.

[64] E. Marquis, D. Seidman, Coarsening kinetics of nanoscale $\mathrm{Al}_{3} \mathrm{Sc}$ precipitates in an $\mathrm{Al}-\mathrm{Mg}-\mathrm{Sc}$ alloy, Acta Materialia 53 (15) (2005) 4259-4268. doi:10.1016/j.actamat.2005.05.025.

[65] S. Iwamura, Y. Miura, Loss in coherency and coarsening behavior of $\mathrm{Al}_{3} \mathrm{Sc}$ precipitates, Acta Materialia 52 (3) (2004) 591-600. doi:10.1016/j.actamat.2003.09.042.

[66] C. Fuller, D. Seidman, Temporal evolution of the nanostructure of Al (Sc, Zr) alloys: Part II-coarsening of $\mathrm{Al}_{3}\left(\mathrm{Sc}_{1-x} \mathrm{Zr}_{x}\right)$ precipitates, Acta Materialia 53 (20) (2005) 5415-5428. doi:10.1016/j.actamat.2005.08.015.

[67] V. B. Shenoy, R. Miller, E. B. Tadmor, D. Rodney, R. Phillips, M. Ortiz, An adaptive finite element approach to atomic-scale mechanics - the quasicontinuum method, Journal of the Physics and Mechanics of Solids 47 (1999) 611-642. doi:10.1016/S0022-5096(98)00051-9.

[68] J. Marian, J. Knap, M. Ortiz, Nanovoid cavitation by dislocation emission in aluminum, Physical Review Letters 93 (2004) 165503. doi:10.1103/PhysRevLett.93.165503.

[69] W. Kim, M. Luskin, D. Perez, A. Voter, E. Tadmor, Hyper-qc: An accelerated finitetemperature quasicontinuum method using hyperdynamics, Journal of the Mechanics and Physics of Solids 63 (2014) 94-112. doi:10.1016/j.jmps.2013.10.001.

[70] H. Moulinec, P. Suquet, A numerical method for computing the overall response of nonlinear composites with complex microstructure, Computer Methods in Applied Mechanics and Engineering 157 (1-2) (1998) 69-94. doi:10.1016/S0045-7825(97)00218-1.

[71] S. Hu, L. Chen, A phase-field model for evolving microstructures with strong elastic inhomogeneity, Acta Materialia 49 (11) (2001) 1879-1890. doi:10.1016/S1359-6454(01)00118-5. 
[72] A. Gaubert, Y. Le Bouar, A. Finel, Coupling phase field and viscoplasticity to study rafting in ni-based superalloys, Philosophical Magazine 90 (1-4) (2010) 375-404. doi:10.1080/14786430902877802.

[73] L. Nguyen, D. Warner, Improbability of void growth in aluminum via dislocation nucleation under typical laboratory conditions, Physical Review Letters 108 (3) (2012) 035501. doi:10.1103/PhysRevLett.108.035501.

[74] V. Bulatov, W. Cai, J. Fier, M. Hiratani, T. Pierce, M. Tang, M. Rhee, K. Yates, T. Arsenlis, Scalable line dynamics in paradis, in: SperComputing 2004, 2004.

[75] L. Brown, Dislocation bowing and passing in persistent slip bands, Philosophical Magazine 86 (25-26) (2006) 4055-4068. doi:10.1080/14786430500501689.

[76] Y. Huang, F. Humphreys, Measurements of grain boundary mobility during recrystallization of a single-phase aluminium alloy, Acta Materialia 47 (7) (1999) 2259-2268. doi:10.1016/S13596454(99)00062-2.

[77] A. Voter, Parallel replica method for dynamics of infrequent events, Physical Review B 57 (22) (1998) R13985. doi:10.1103/PhysRevB.57.R13985.

[78] M. Sørensen, A. Voter, Temperature-accelerated dynamics for simulation of infrequent events, The Journal of Chemical Physics 112 (21) (2000) 9599-9606. doi:10.1063/1.481576.

[79] A. Laio, F. Gervasio, Metadynamics: a method to simulate rare events and reconstruct the free energy in biophysics, chemistry and material science, Reports on Progress in Physics 71 (12) (2008) 126601. doi:10.1088/0034-4885/71/12/126601.

[80] D. Sheppard, R. Terrell, G. Henkelman, Optimization methods for finding minimum energy paths, The Journal of Chemical Physics 128 (13) (2008) 134106. doi:10.1063/1.2841941. 